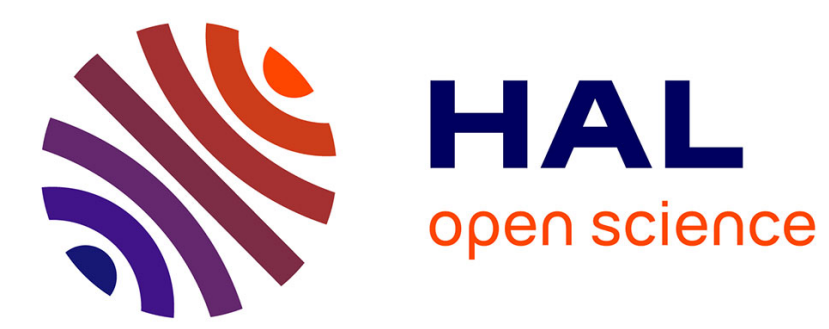

\title{
Imperator Caelestium
}

Gilbert-Charles Picard

\section{To cite this version:}

Gilbert-Charles Picard. Imperator Caelestium. Gallia - Fouilles et monuments archéologiques en France métropolitaine, 1977, 35 (1), pp.89-113. 10.3406/galia.1977.1557 . hal-01939329

\section{HAL Id: hal-01939329 \\ https://hal.science/hal-01939329}

Submitted on 14 Jun 2020

HAL is a multi-disciplinary open access archive for the deposit and dissemination of scientific research documents, whether they are published or not. The documents may come from teaching and research institutions in France or abroad, or from public or private research centers.
L'archive ouverte pluridisciplinaire HAL, est destinée au dépôt et à la diffusion de documents scientifiques de niveau recherche, publiés ou non, émanant des établissements d'enseignement et de recherche français ou étrangers, des laboratoires publics ou privés.

\section{(이) $\$$}

Distributed under a Creative Commons Attribution - NonCommercial - NoDerivatives| 4.0 


\title{
IMPERATOR CAELESTIUM ${ }^{1}$
}

\author{
par Gilbert Charles PICARD
}

bans son catalogue des dieux gaulois, César nomme un maître des chosés célestes; il l'appelle Jupiter, bien que la place qu'il lui attribue dans l'énumération, qui est l'avantdernière, incite à penser que ce Jupiter celtique n'avait pas dans son panthéon la mème: prééminence que son homologue romain dans le sien². Les doruments postérieurs à la ronquête montrent Jupiter, tantòt sous l'aspect de Zeus, tantòt avec une apparence originale. Ainsi quelque cent soixante groupes, retrouvés sur le territoire des Giaules et des Germanies romaines, représentent un dieu cavalier que supporte un anguipede couché3 (fig. 1); les dédicaces qui areompagnent certains de ces monuments nomment Iupiler Oplimus Maximus ; un certain nombre de ces statues ont d'ailleurs conservé un foudre que brandissait le dieu. La majorité de ces groupes étaient portés par une colonne, souvent surélevée sur deux piédestaux, l'inférieur grénéralement quadrangulaire, le supérieur ordinairement octogonal. Cas piédestaux portent sur leurs faces la représentation de divinités qui ont valu aux quadrangulaires - ainsi qu'à des autels dont il est quelquefois difficile de les distinguer le nom de "pierres à quatre dieux" (Viergöttersteine)4.

Les nombreux savants qui se sont intéressés à ces monuments depuis la fin du siècte dernier, ont essayé d'abord de déterminer avee le plus de précision possible les données fondamentales suivantes:

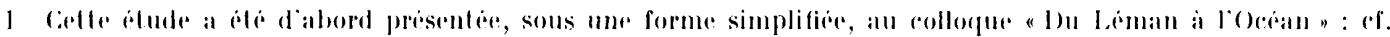
Cicesarodunum, 10, 1975, p. 176-181.

2 B.(i., VI, 17, 2. Post hunc (Mercurium), Apollinem el Vurtem el Ionem el Vinervam. Le his eadem fere quam

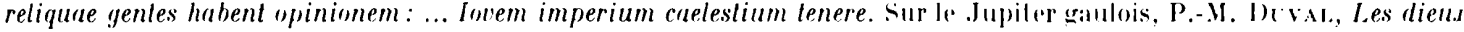

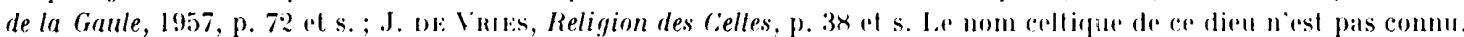

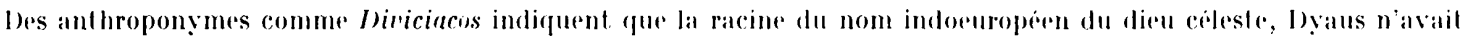

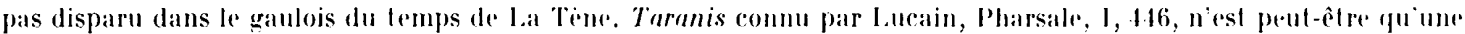
ipithete designant te dieu du tonnerre.

3 La hibliographie sur le dieu cavalier à l'hippophore est enorme, mais le seul ouvrage d'ensemble reste celui

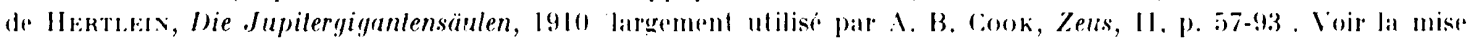
all point de P.-.ll. Drvat, dans Rayonnement des cirilisalions grecque el romatine... idetes du VIIJe Congries

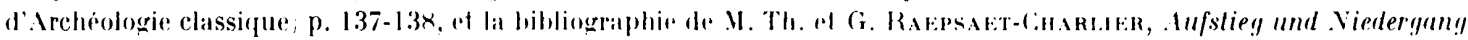
der Rïmischen Well, $11,1,1975, \mathrm{p} .211$.

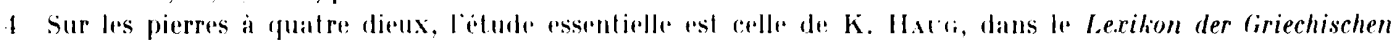

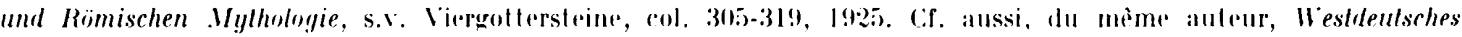
Zeilschrifl, 10, 18:1, p. I0 a s. 


\begin{tabular}{|c|c|c|c|c|c|c|c|}
\hline Catégorie & Série & Groupe & $\begin{array}{l}\text { Nature des } \\
\text { monuments }\end{array}$ & Nombre & Localisation & Chronologie & Observations \\
\hline 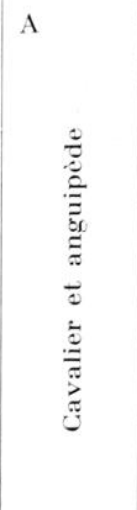 & 2 & b & $\begin{array}{l}\text { colonnes sur double } \\
\text { piédestal suppor- } \\
\text { tant un groupe de } \\
\text { Jupiter cavalier et } \\
\text { de l'hippophore } \\
\text { colonnes dépour- } \\
\text { vues d'un élément } \\
\text { groupes cavalier et } \\
\text { hippophores non } \\
\text { portés } \\
\text { figurines }\end{array}$ & $\begin{array}{c}\text { env. } 160 \\
\text { groupes } \\
\text { entre } \\
250 \text { et } 300 \\
\text { pierres à } 4 \\
\text { dieux } \\
?\end{array}$ & $\begin{array}{c}60 \% \text { entre } \\
\text { Meuse et } \\
\text { Rhin } \\
20 \% \text { à l'e. } \\
\text { du Rhin } \\
7 \% \text { b. Seine } \\
7 \% \text { b. Loire } \\
\text { ? }\end{array}$ & $\begin{array}{c}\text { entre } 50 \\
\text { et } 250\end{array}$ & $\begin{array}{l}\text { multiples varian- } \\
\text { tes : il n'y a pas } \\
\text { deux monuments } \\
\text { exactement sem- } \\
\text { blables. } \\
\text { un seul exemple } \\
\text { sur : colonne de } \\
\text { Cussy. }\end{array}$ \\
\hline 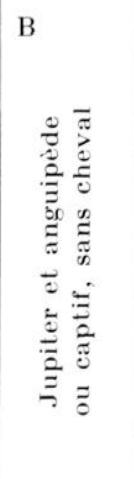 & 1 & & $\begin{array}{l}\text { Jupiter debout po- } \\
\text { sant sa main sur la } \\
\text { tête d'un anguipède } \\
\text { dressé contre sa } \\
\text { jambe } \\
\text { Jupiter debout, cap- } \\
\text { tif barbare à son } \\
\text { côté } \\
\text { Jupiter enjambant } \\
\text { l'anguipède } \\
\text { Jupiter assis, angui- } \\
\text { pède à ses pieds }\end{array}$ & $\begin{array}{c}5 \text { groupes } \\
\text { env. } \\
10 \text { figurines } \\
1 \text { stèle } \\
5 \text { groupes } \\
2 \text { groupes } \\
1\end{array}$ & $\begin{array}{l}3 \text { groupes en } \\
\text { Aquitaine, } 2 \\
\text { en Rhénanie } \\
4 \text { en Aqui- } \\
\text { taine, } 1 \text { en } \\
\text { Rhénanie } \\
\text { Grand et } \\
\text { Nimègue } \\
\text { Mouhet } \\
\text { Indre) }\end{array}$ & $\begin{array}{c}\text { I }^{\text {re }} \text { s. ou } \\
\mathrm{II}^{\mathrm{e}} \\
\mathrm{II}^{\mathrm{e}} \mathrm{S} . \\
? \\
\mathrm{II}^{\mathrm{e}} \mathrm{s} .\end{array}$ & $\begin{array}{l}\text { l'anguipède des } \\
\text { figurines est femelle } \\
\text { parenté étroite } \\
\text { avec statues d'Ha- } \\
\text { drien et Antonin } \\
\text { la restitution du } \\
\text { groupe de Nimègue } \\
\text { est problématique }\end{array}$ \\
\hline 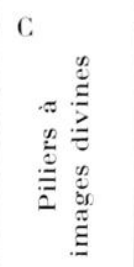 & 1 & & $\begin{array}{l}\text { piliers avec images } \\
\text { de dieux celtiques } \\
\text { et romains } \\
\text { piliers avec images } \\
\text { de dieux romains }\end{array}$ & 2 & $\begin{array}{l}\text { Paris et } \\
\text { Mavilly } \\
\text { Cologne et } \\
\text { Vinsobres }\end{array}$ & $\begin{array}{l}\text { 1re moitié } \\
\text { du } I^{\text {er }} \text { s. } \\
\text { II }^{\text {e }} \text { s. ? }\end{array}$ & $\begin{array}{l}\text { certaines pierres à } \\
\text { quatre dieux pour- } \\
\text { raient appartenir à } \\
\text { cette série }\end{array}$ \\
\hline 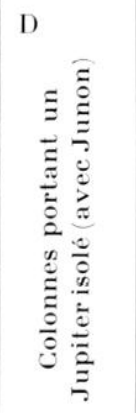 & $\begin{array}{l}1 \\
2\end{array}$ & $\mathrm{~b}$ & $\begin{array}{l}\text { colonne de Néron } \\
\text { colonnes portant un } \\
\text { Jupiter trônant } \\
\text { portées sur pierre } \\
\text { à } 4 \text { dieux } \\
\text { avec images sur le } \\
\text { fût } \\
\text { mosaïque représen- } \\
\text { tant le culte d'une } \\
\text { statue jovienne sur } \\
\text { colonne }\end{array}$ & $\begin{array}{c}1 \\
12\end{array}$ & $\begin{array}{l}\text { Mayence } \\
\text { Germanie } \\
\text { inférieure } \\
\text { St-Romain- } \\
\text { en-Gal }\end{array}$ & $\begin{array}{c}? \\
? \\
\text { vers } 200\end{array}$ & \\
\hline 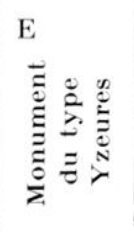 & & & tour à étages & 3 au moins & $\begin{array}{l}\text { Aquitaine } \\
\text { (Turons, } \\
\text { Pictons, } \\
\text { Bituriges) }\end{array}$ & fin $11^{e} s$ & $\begin{array}{l}\text { certaines pierres à } \\
4 \text { dieux apparte- } \\
\text { naient probable- } \\
\text { ment à des monu- } \\
\text { ments de ce type }\end{array}$ \\
\hline
\end{tabular}



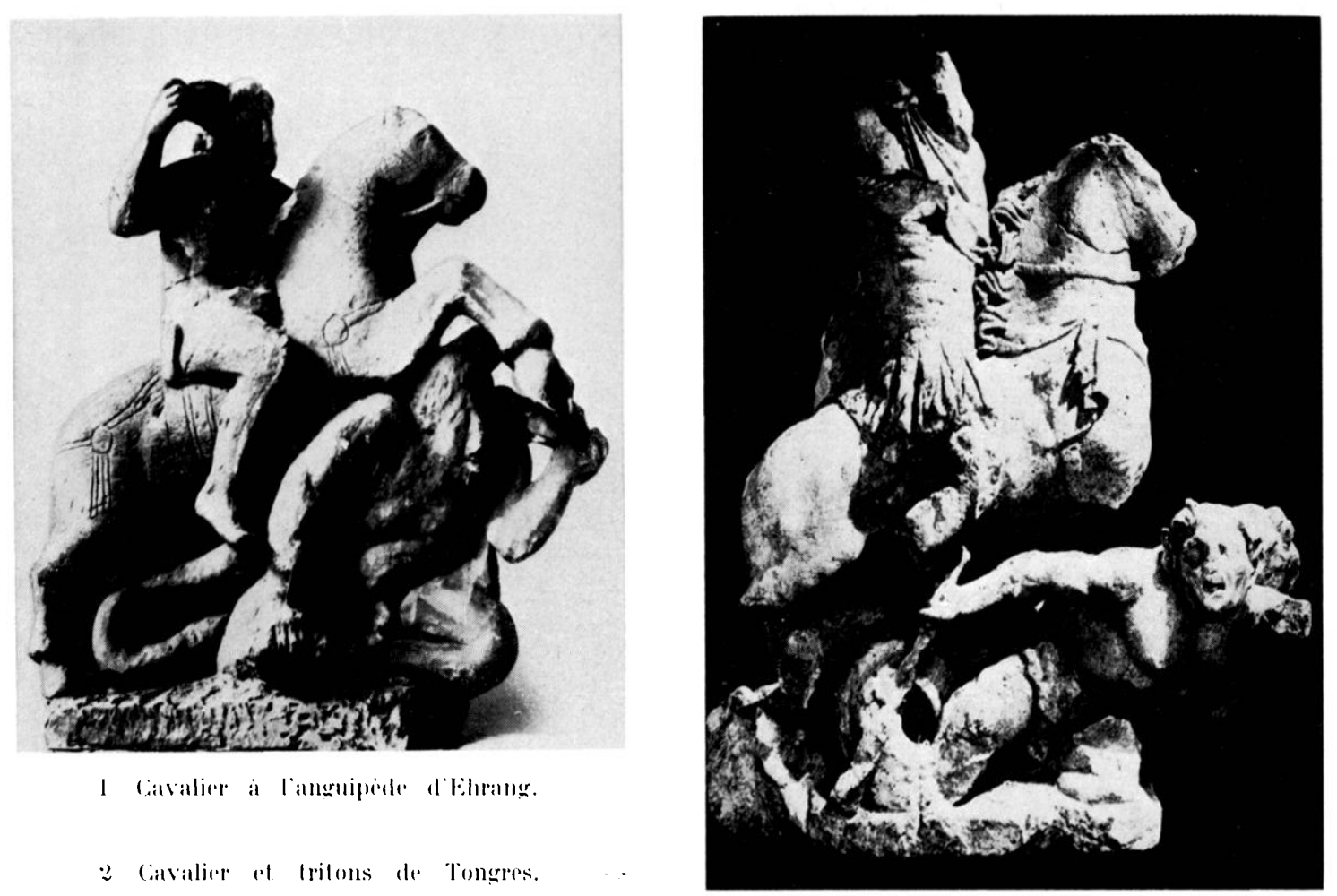

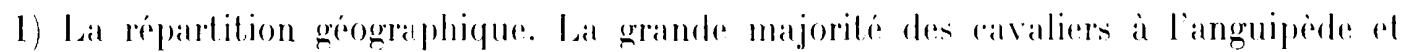
des pierres a quatre dieux provient de l'lest de la baule et des diemanies romaines, y

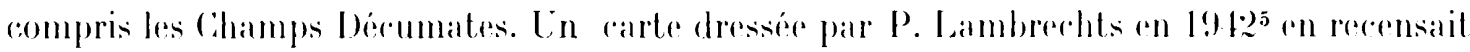

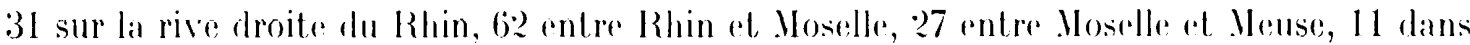
le bassin de la seine, ? sur la rive droite de la lodere. 3 an Armorigue, 6 anu sud de la Loire; on en ronnait un en Angleterre, pres de Cambridge. Il n'an existe ancem dins une autre

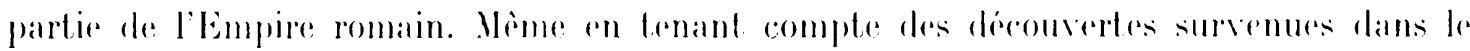

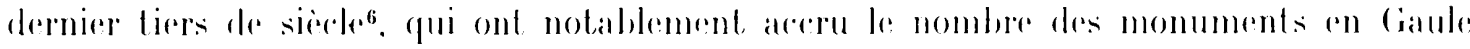

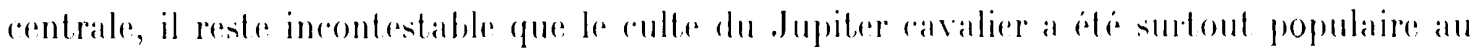
voisinage du limes rhénan, mais qu'un nombere non négligeable de ses monuments se rencontre sur le reste du territoire gallo-britamnigue.

2) Ia chronologie des documents. I'n certain nombere de colonnes de Jupiter sont latées épigraphiquement. L a plus ancienne remonte a l'an 170 de notre ère, la plus récente

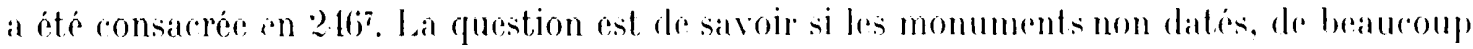

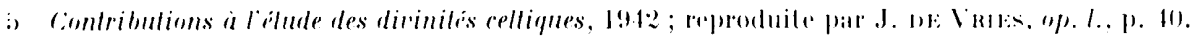

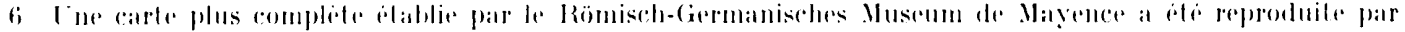

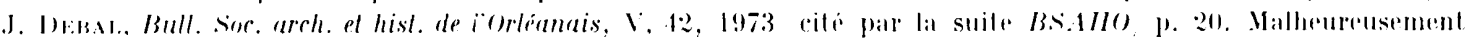

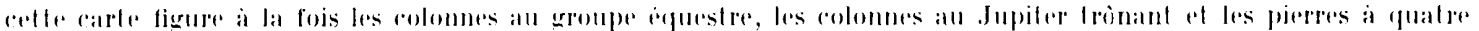

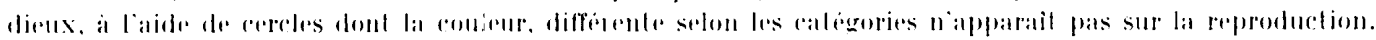

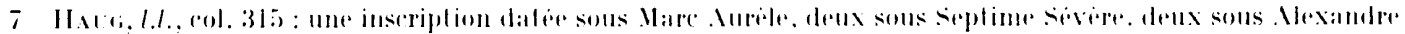

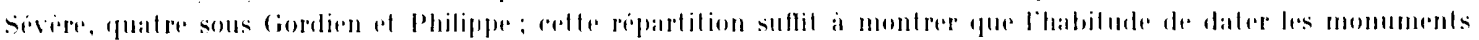

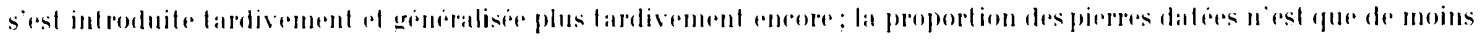
do 50 , dutulat. 
les plus nombreux, se situent tous dans cette tranche chronologique. (on n'y peut guère: répondre, en equ qui concerne les monuments anciennement découverts, que par l'analyse stylistique; nous essilerons de le faire, provisoirement et partiellement, dans la suite de ce travail. I)ans le cas rarissime de la découverte nouvelle d'un mourement qui n'a été déplacé ou réutilisé ni dans l'Antiquité tardive ni au Ioven Îge. les données archéologiques fournissent un excellent critere de datation; cest ce qui s'est produit récemment a Tongres (fig. 2$)^{8}$; or, J. Nertens ronclut dans ce cas à une date flavienne, antérieure done d'une: centaine d'années à la plus ancienne dédicace épigraphique. Au contraire, il n'existe aurune raison de penser, avec (i. Becatti, que des colonnes aient été dressées après les invasions du III $^{\mathrm{e}}$ siècle ${ }^{9}$.

3) Rapports entre les colonnes de Jupiter et d'autres monuments. On a souvent considéré comme apparentés aux colonnes de Jupiter des monuments d'une autre catégorie: ainsi le pilier des nautes de Paris est dédié à Jupiter et les dés superposés qui le constituent peuvent ètre considérés comme autant, de "pierres à quat re dieux"

Cne partire considerable des études est ronsacrée à la signification des monuments. à leurs liens avere une population déterminére il s'agit là. nous semble-t-il, de considérations qui ne devatent itre aborders qu une fois resolus les problemes fondamentaux de répartition géographique, de typologie at de chronologie. Or. resux-ci sont evidemment étroitement imbriques, at le plus important concerne la typologie ; on ne peut en effet etudier les répartitions dans le temps et dans l'espare que si on sait exartement quels monuments il s'agit de clisser. Vous avons pu constaler que cettre typologie avait été insuffisamment traitie : des 15 lypes qui vont atre examinés, quatre navitent jamais été pris en conside-

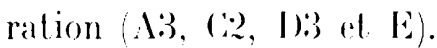

Nous rlassons dans la categorio $A$ tous les monuments comportant la representation du calvalier à languipede.

La série .11 romprend les colonnes de type "canonique". c'est-ii-dire celles qui comportent le double piédestal, le fint, le chapiteau, el le groupe du cavalier et de l'hippophore. Cette série est de beaucoup la plus nombreuse (plus de 100 monuments). Hais elle présente des variations portant sur tous les éléments du monument:

a) le dieu cavalier peut être imberbe ou barbu. nu, vêtu (de manière fort diverse), cuirassé, et porte des attribuls divers (foudre, roue ou lance). La tête est nue ou couronnée de lauriers (a (irand).

b) La monture est généralement un cheval, harnaché (avec une selle ou avec un tapis) ou nu. Il charge au galop ou marche au pas. Dans un cas, Jupiter est en char11.

c) L'hippophore est généralement un personnage anguipicde unique, et autant qu'on puisse en juger, mâle (figr. 3). Mais on connaît des angruipèdes femelles ${ }^{12}$. Lu lieu d'anguapèdes on peut trouver

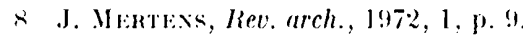

9) La colonna coclide isloriala, p. 13-1.1.

10 Lointegration dans une meme calegorie du pilier des nautes et des colomnes an cavalier a ble sontenute

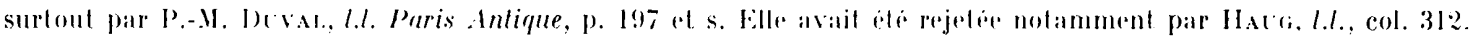

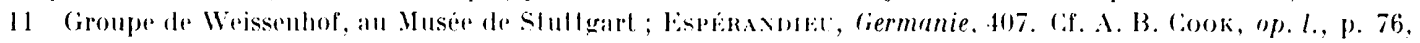
fig. 36 .

12 A Saint-Maho en Bretagne. Esp., 3036. C.f. J. ne Vrtis, op. I., p. 41. 11 existe des chapiteaux decorés

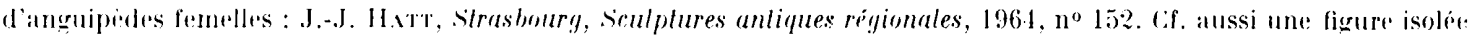

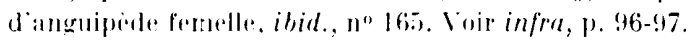


des Tritons ${ }^{13}$. I Tongres ce sont deux géants que renverse la charge impétueuse de Jupiter. I Vienneen-Val, l'hippophore. peul-îlre acroupi dans la pose ordinaire de "Cermunnos "lt. esl flanque de deux petils assesseurs qui soutiennent les pieds du cavalier. Dans un groupe de Luxeuil connu seulement par des dessins du xime siecle ${ }^{15}$, le cheval au pas pose le pied sur la tète d'un personnage enterré jusquau cou. C'est a Lort qu'Espérandieu a suspecte l'exaclitude de cette reproduction : une statuette de la sirie BI provenant de Iompierre-les-Eglises'16 montre en effet. Jupiler debout

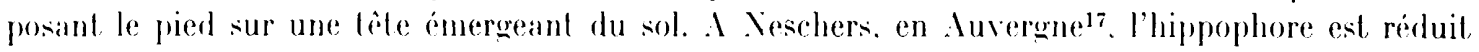
a un masque. riganlesque par rapport au ravalier, pourvu de deux bras qui sont soudés a son orciput. Il es lacile de trouver l'origine de ces représentations dans l'art impérial. Ia slatue équestre de Iomitien dresse au Forum Romain en 9l nous est connue par des monnaies el par le premier poime des Silres de slare : l'empereur étail représenté sur un cheval au pas qui posait le pied sur la teite du Rhin raptif. imergeant du sol:

\section{racuate per caespile lerrae aerea captiui crinem legil ungula Rhenis}

las statue de luxeuil devait reproduire assez fidedement le modele romain. Celle de Veschers apparal. comme nne araptation inspirée par l'imagination fantastique d'un sculpteur encore pénétré d'esprit reltique ${ }^{19}$. Le symbolisme en tout ras est relui mime qu'indique stares : Jupiler fou Inomitien) Iromphent sans combaltre (Domitien garde l'épée au fourreau) par la seule manifestalion de leur "sacré ". Le lleure. ou le génie rhthonien ne peut s'opposer a relte force surnaturelle, mais r'est malgré eux, en vancus el raptifs. quils la subssent. On souleve donc un faux probleme quand on rherche a distinguer des hippophores lult ant cont re leur vainqueur el des hippophores "collaborant" avec lui ${ }^{20}$. I a distinction entre les deux rasses n'existe qu'en fonction d'une conception théologique hellénistico-romaine du pouvoir de la Providence. considérée tantòt romme transcendantale et Lantòt comme militante.

d) Le chapiteau. Cerlaines colonnes au cavalier avaient un chapiteau non figuré21 mais la grande majorilé possiole un chapiteau corinthisant, généralement orné sur chaque face d'un buste féminin qui a été interprété soit comme une siason soit comme une heure du jour ou l'un des âges de la vie ${ }^{22}$. Ces chapiteanx corinthisants sc répartissent en quatre rlasses:

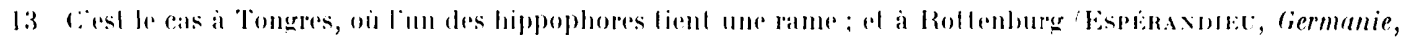

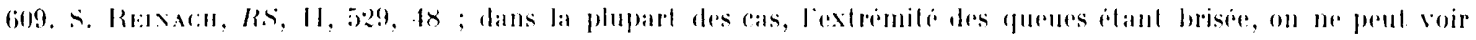

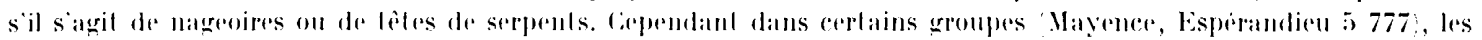
serpents mordent la diem.

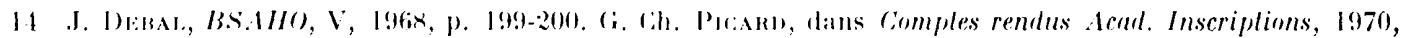
p. $17 !$.

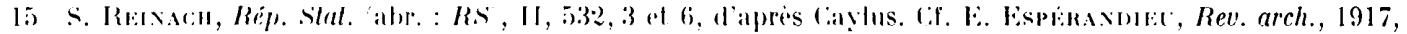
1, 1. 75 .

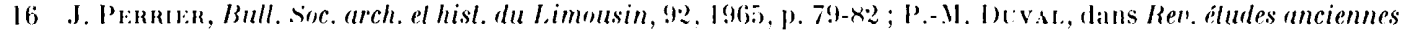

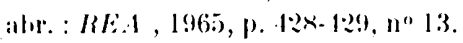

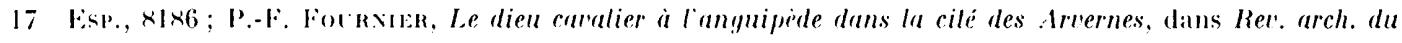

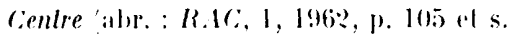

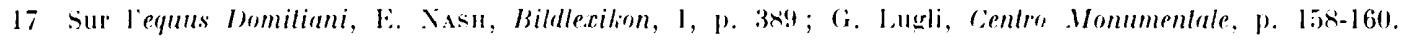

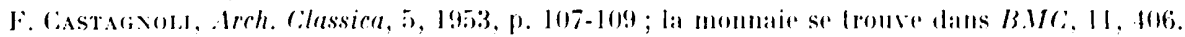

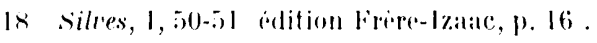

19 I. dessin de Caylus fait apparaitre, du cole gauche du cavalier, un personnage ren chlamyde que le dien

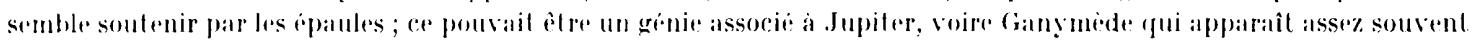

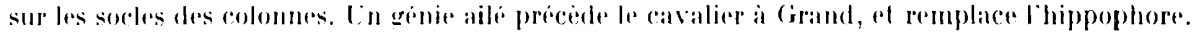

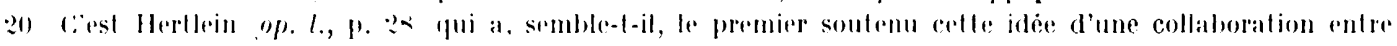

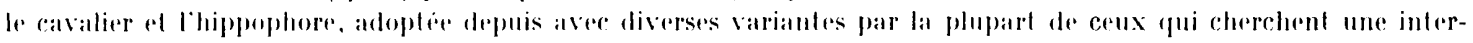

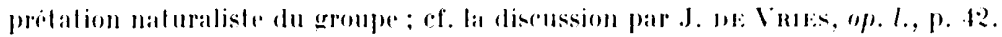

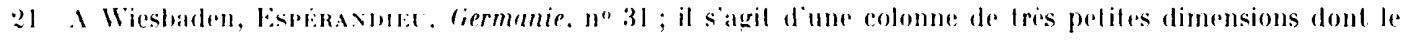
lambour n'est pas sculple.

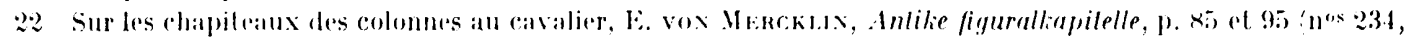

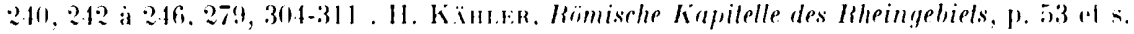




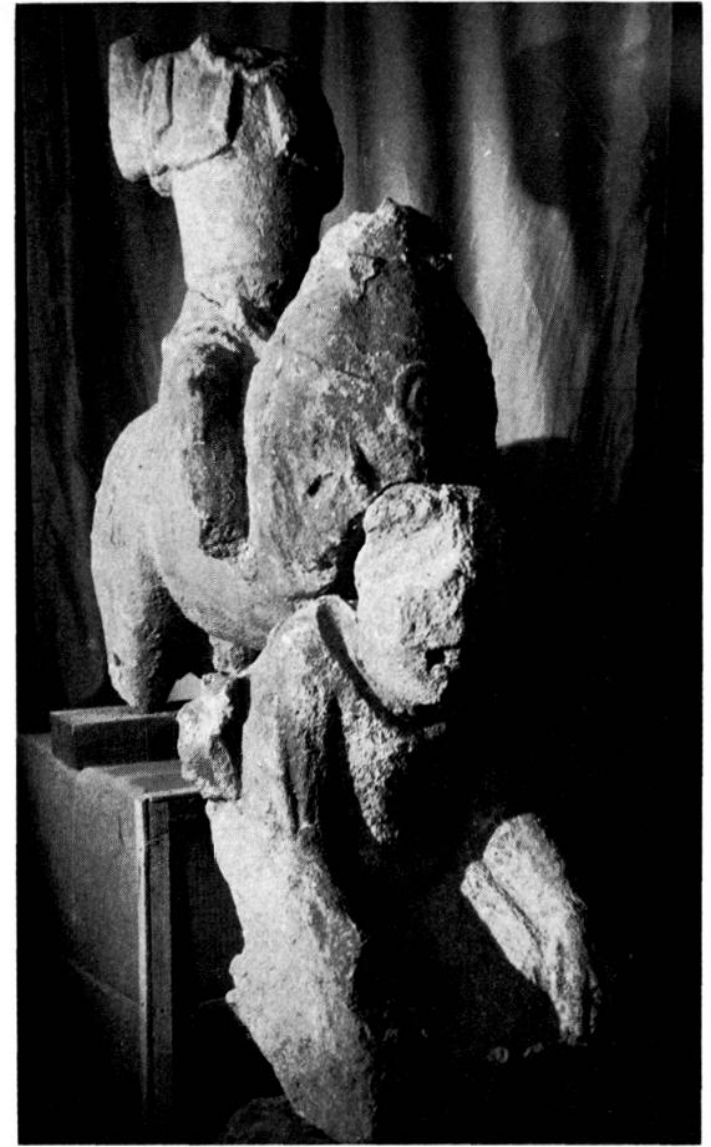

3 Cavalier et hippophore de Vienne-en-Val.

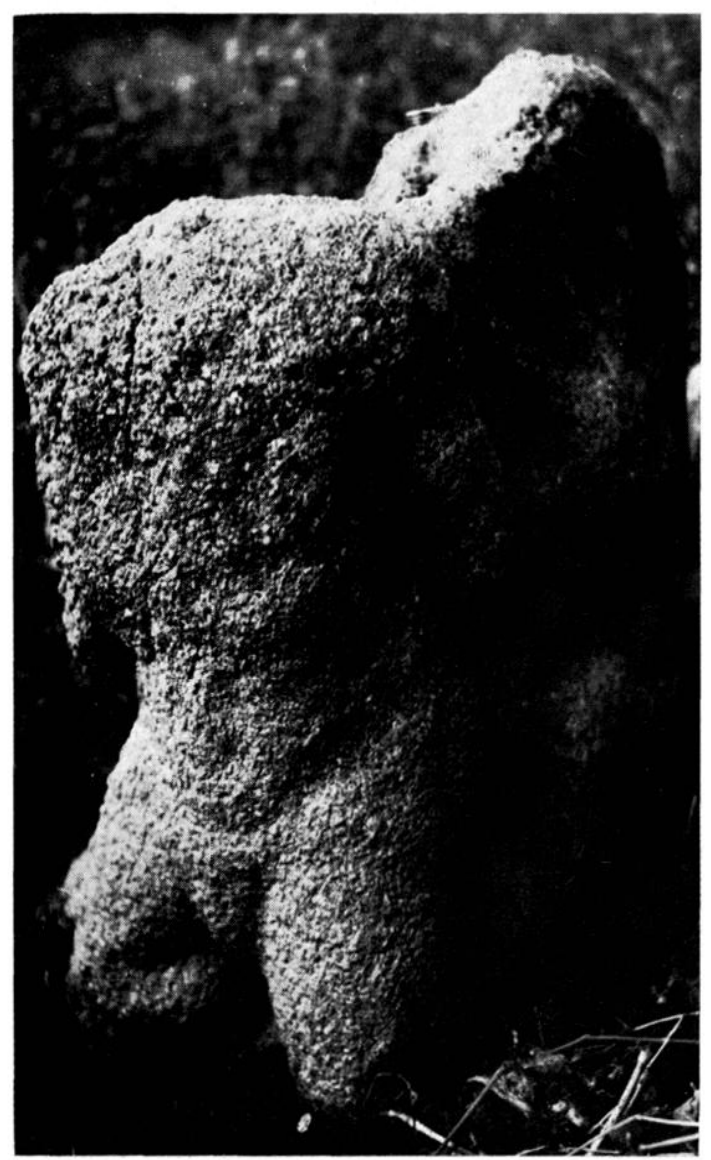

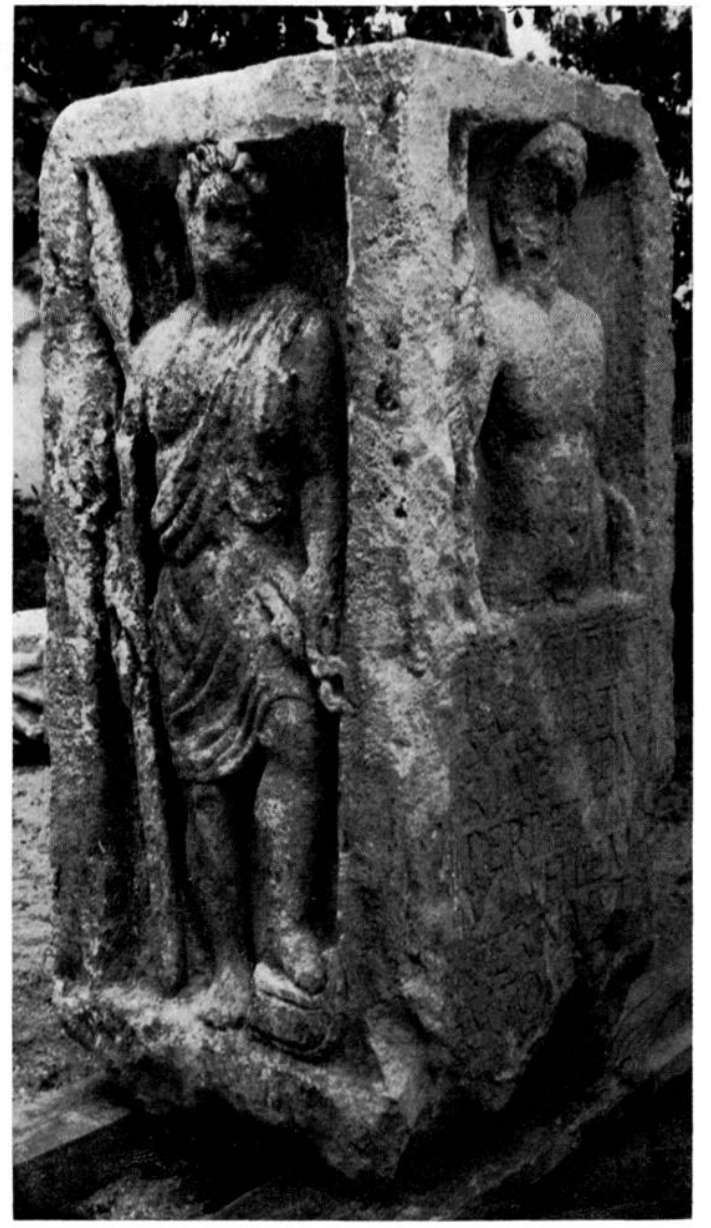

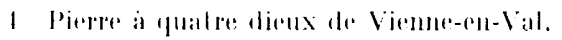




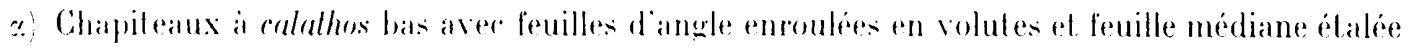
au milieu de rhange fare el portanl un husle. Ce type derive diun modele ilalo-hellénistique bien connu, representio en cianle à Cilanum.

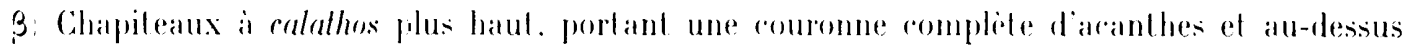
un hustr emearler par les leuilles dangle en volutes. Cóst le lype le plus répandu.

y Chapiteaux a double couronne d'aranthes al a volutes nomales entre lesquelles se detache le buste.

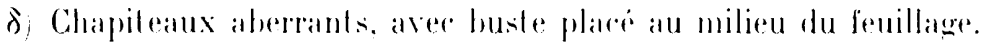

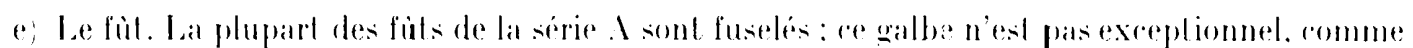

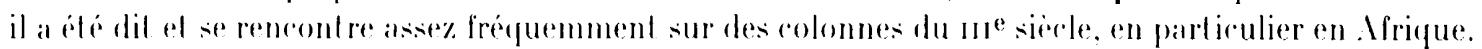

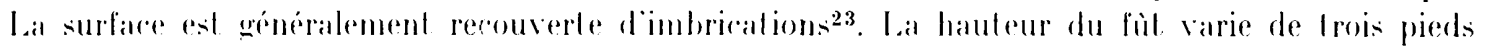
(Wiestarden à plusieurs mitres.

f) Ta base est lantol de type allique el lantot de lype tosrant.

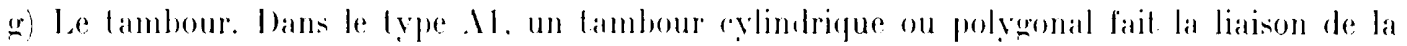
eolonne el du piedestal. Ce lambour peut alre lisse2t mais le plus souvent il porte, dans des niches, les images des dieux de la semaine. completeses dans le cis d'un lambour aclogonal, par une figure supplementaire. Vais on trouve anssi des dieux on genies lress divers. y rompris, semble-t-il. dans III ras. les génies personnels des dédicants ${ }^{25}$.

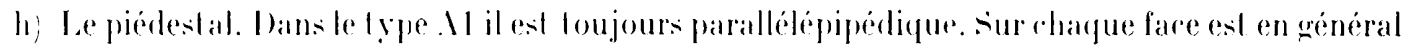

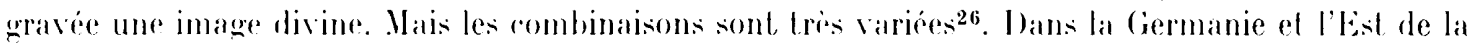
Giale. environ bo ${ }_{0}$ des liergältersteine présentent Junon. Minerve. Hercule et Mercure.c'est-i-dire les deux compagnes de Jupiter dans la triade capiloline associesesu plus populaire des dieux romanogermaniques ef au premier des dieux gallo-romains. Dans le resle de la (ianle nous rencontrons des formules tres diverses of notamment. a Paris el a Vienne-en-Val (lig. 1). un groupement Mars, Vénus, Vulain, hérité peut-ìlre d'une devotion résariennez2

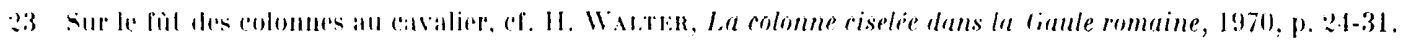

21 .1 Mirstiallon, supra, II. 2:2.

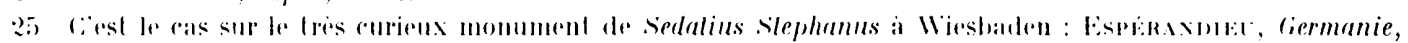
111 ; infra. 1). 111 .

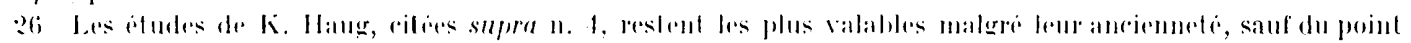

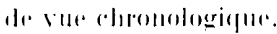

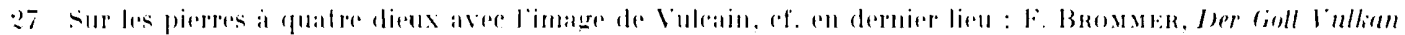

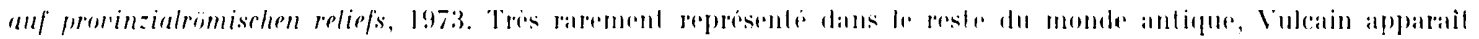

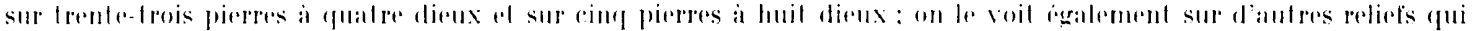

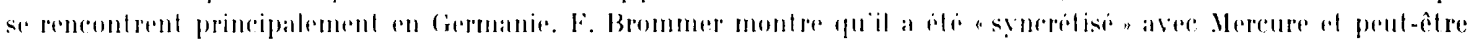

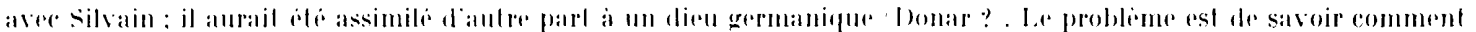

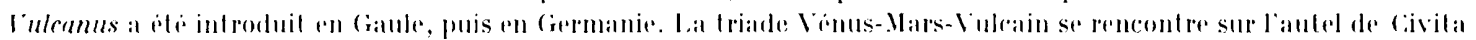

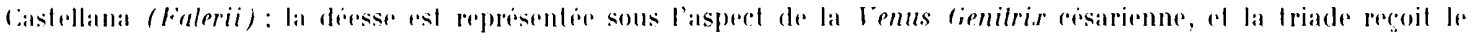

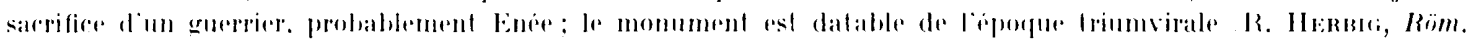

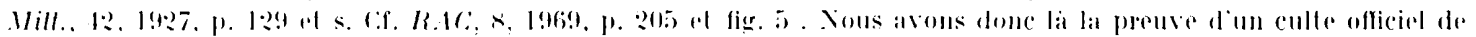

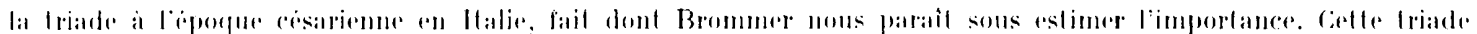

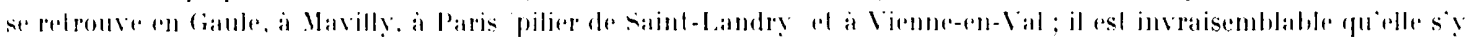

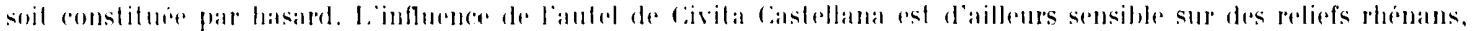

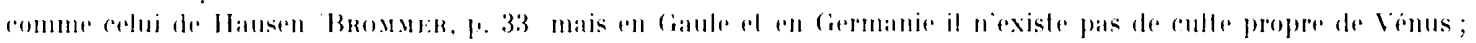

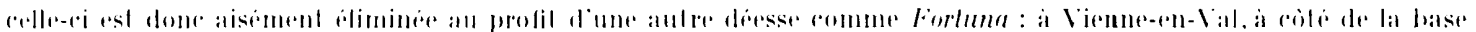

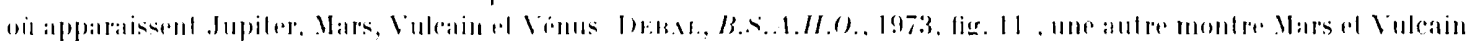

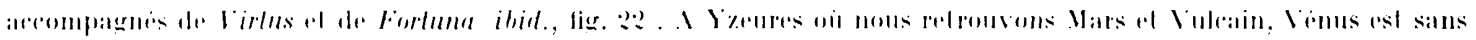

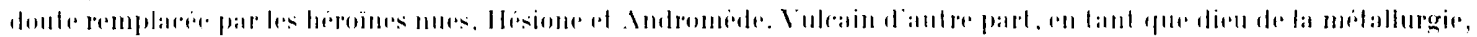

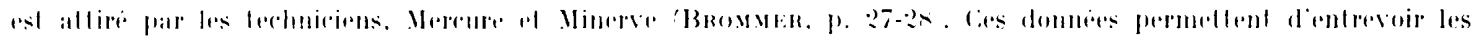

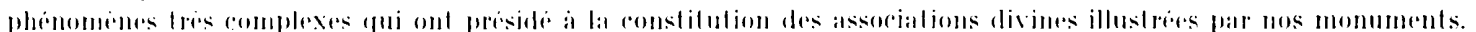

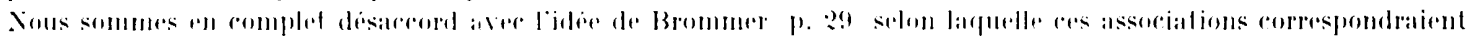

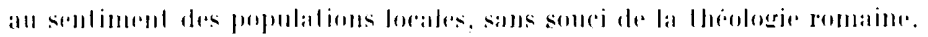


Xous placons dans la serie 12 des colonnes auxquelles manque un des éments de la sirie 11. La cólebre colonne de Cussy dans la Cole-d'or n’a qu'un piédestal octogonal. sculpte des images de Junon. Minerve, Hercule. Vulcain, quareompagnent Mercure?. Veplune, une nymphe lees deux dernieres figures indiquant sans doute le lien aver un culte de source ansi que Gianymede. l ne image de Jupiter couronnait surement le monument, mais il n'est pas prouré qu elle ait ete equestre?8.

Sous rangeons dans la série $1: 3$ a des images du cavalier a l'hippophore qui n'ibaient pats exhaussees sur colonne : c'elait le cas. fort probahlement, du principal groupe de Vienme-en-Val29. I'aul res groupes du mème lyge étaienl sans doute presentes allsis sur une base peu elevée. mais il est difficile de les identifier. En lout ars l'existence de res sculptures est rendue cert aine par relle de représentations miniatures en terre cuite, qui forment notre groupe $1: 3$ b ; res ligurines sont peu nombreuses : on n'en connait que deux. dont une appartient au Musée des antignilés nationales a

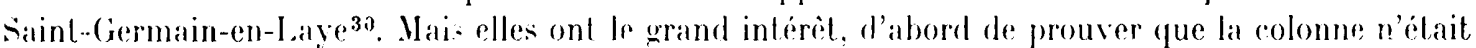
pas le support indispensable du lype, et que par conséquent il n'en faut pas tenir compte pour déterminer sa signification ${ }^{31}$; ensuite de confirmer qu'il s'agisisal d'une forme cultuelle dont le rayonnement dépassail un cadre strictement régional.

La catégorie B groupe les Jupiters associes à un anguiperle, mâlr ou femelle ou à un captif, mais non montes : elle comprend quatre siories :

B1. Le dieu est deboul. l'anguiperde plus petil dresse contre une de ses jambes, la main du dieu posée sur sa chevelure, dans un geste de domination, et non de communion comme l'avait cru F. Benoit ${ }^{32}$. Cetle série est représentée par un groupe en ronde bosse inédit. assez bien conservé, ¿ Argentomagus (Biluriges Ciubi). les débris plus ou moins mutilés de qualre autres a Vayres (Gironde) et a Bordeaux, chez les Biluriges Cievisci, a Ladenburg ef a Rottweil en Ciermanie : en outre, une dizaine de figurines du Vusée des antiquités nalionales, fabriquées dans l'Allier a celles-ci présentent des particularités remarquables : l'anguipede est femelle, le dieu porte des braies l ne sle de découverte a Reze (Loire- Mlantique), chey les Namneles représente dans une niche un dieu imberbe posant la main sur la tête d'un anguiprede grimaloant33.

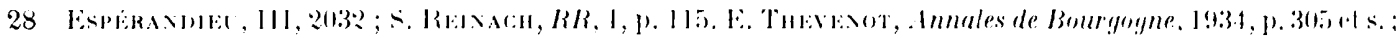

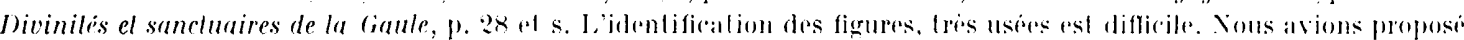

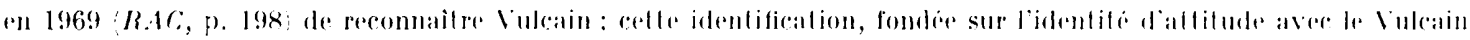

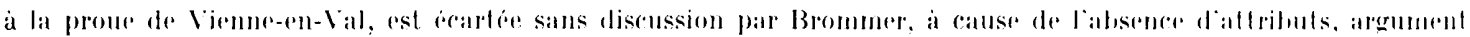

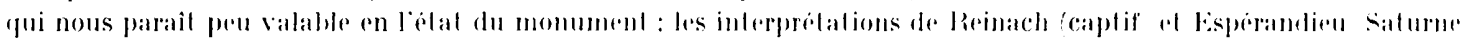

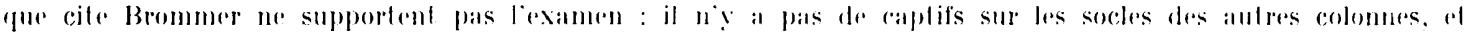

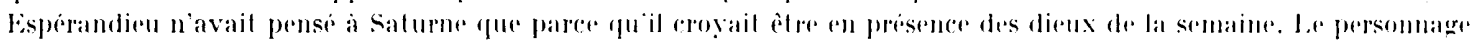

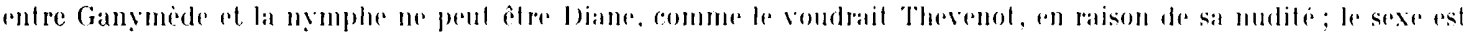

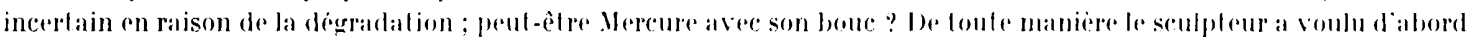

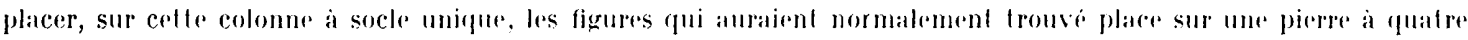
dieux : Junon, Vinerve el Hercule : Vulcain complete hien cetle serie dans laguelle Vercure soinsere aussi naturellement.

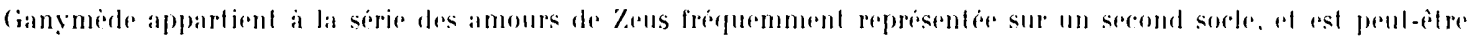

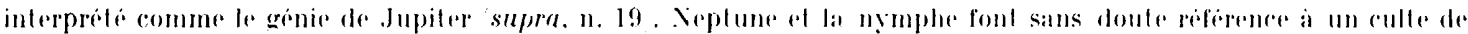

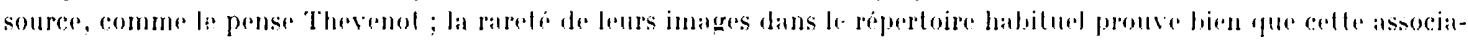
tion diune somere ef d'une colonne n'itait quirexeptionnelle.

29 J):BAL, B.S.1H(), 1973, p. 13-14.

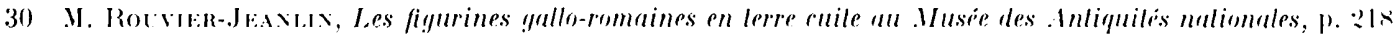
a pl. no 522

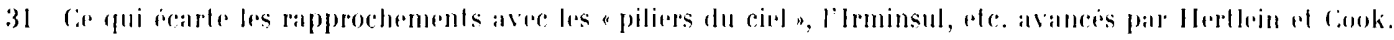

32 Milanges Charles Picard, I, p. 52.

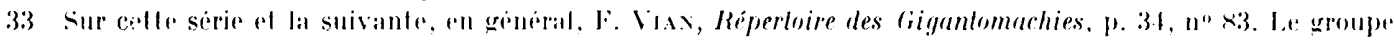

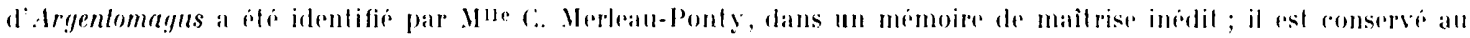

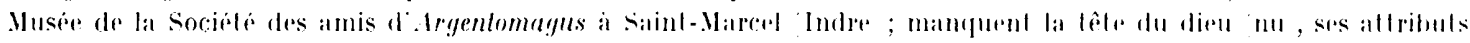

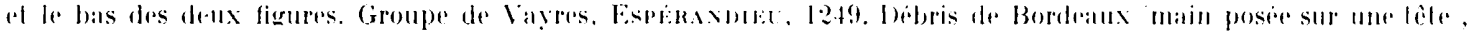

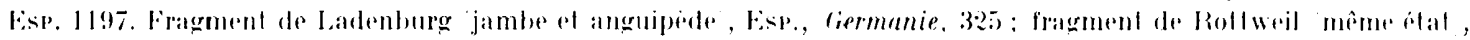

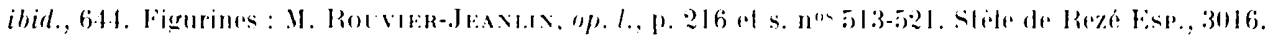


B2. Cette série ne differe de la précedente que parce que l'anguiperde est remplare par un humain enchaine. de type barbare. Elle comprend un groupe en ronde bosie presque complet, Irouvé a saint-Christophe-le-Chaudry, ches les Biburipes Cub: : un second. auquel ne manque que

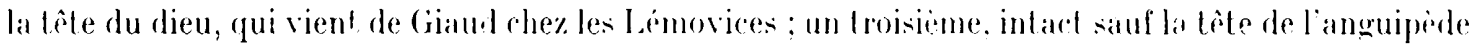

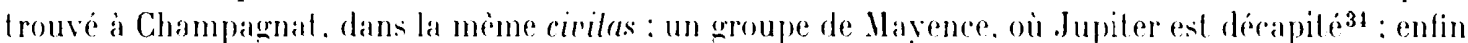

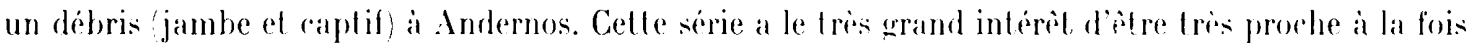
de la précédente. aver qui elle a souvent éte confondue, el doune série de statues impériales ayant uil captif à leur pied ; les plus connues sont les Harbiens du type de Hierapytnat ef rette famille est représentée en Gaule par l'Intonin du theitre d'(orange ${ }^{35}$. I semble bien. d'ailleurs. que le Jupiter de sinint-Christophe-le-Chaudry ait un visage asse\% proche de relui d'Hadrien ou d'Antonin.

B3:3. Celte serie ne romprend qu un monument : un groupe de Girand. alu Musce d'Epinal: le dieu. décapité, enjambe une anquiperde femelle ${ }^{36}$. On sail que le sancluaire de Cirand. ou Apollon étail le dieu principal. a rendu un groupe. dailleurs aberranl. de la ratégorie . A. série 1. Jupiler devait être la l'hole d'Apollon. comme Apollon était 'hòte de Jupiter a Vienme-en-Val.

B1. Cette série ne comprend alussi qu'une pièe : le groupe du Mouhet Indre) (fig. ol. non loin d'Argenlomagns ${ }^{37}$. Jupiter est représenté assis, sur un sioge dont le dosicer est décoró diune roue fet non sur un char, car la roue unisue est sculptée sur la face postérieure et non sur une face latrrale): un anguiperde aux mains ligotées est agenouille sur le colé qauche du siege; l'un des pieds du dieu est posé sur l'une de ses "jambes: serpentines.

La ratégorie C comprend des piliers quadrangulaires ornes d"umages divines ; cerlaines de ces figurations sont d’inspiration purement celtique ; elles apparaissent sur des monuments dont l'un, le pilier des nautes de Paris, est par chance date du regne de Tibere par son inscription.

Il constituera notre série C1 avec le pilier de Vavilly ${ }^{38}$. Ie problime posé par ces documents est celui, envisagé ci-dessus de leur parenté avec les colonnes de la série A1. Nous avons donné les raisons qui permettent d'affirmer celte parenté pour le pilier de Paris; relui de Mavilly, dont on ignore le dédicataire. est en tout cas formé par la superposition de pierres à quatre dieux ${ }^{39}$.

La série Ci2 comprend des piliers éçalement quadrangulaires exclusivement ornés d’images de divinités classiques. In pilier du musée de Cologne, provenant de Ceumarkt ${ }^{40}$, porte les images superposées de Vénus, Vulain el Minerve. A Vinsobres, press de Nyons, fut découvert en 1971 un pilier de section rarrée portant les images, elles aussi superposées. de Junon el de Minerve41. Jans le premier cas nous avons donc une désse capitoline associée a deux membres de la "lriade césarienne" déja rencontrée dans la série $\mathrm{M1}$; dans le second, deux déesses apilolines. Ia série Ciz.

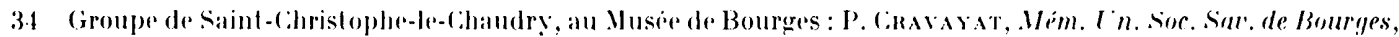

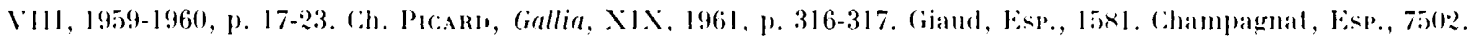
Mayence, Esp., 5772. Andernos, Jsp., 1237.

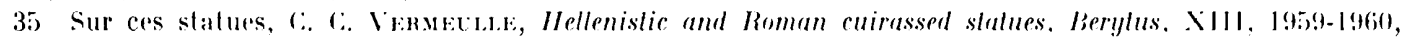

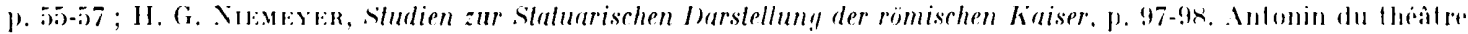

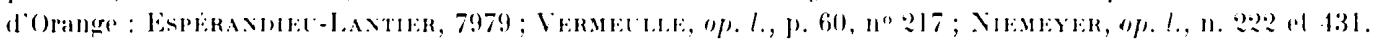

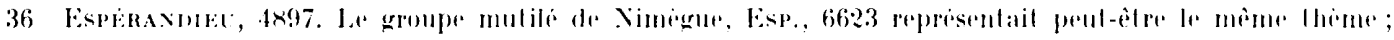

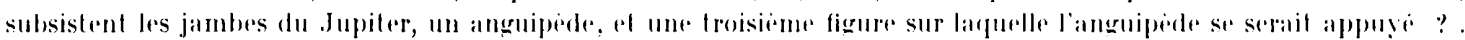

37 Ch. I.tolowi, dans R.A6, IX, 1976, p. 1:3-1:6.

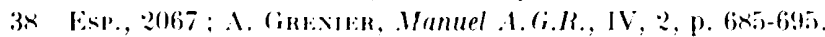

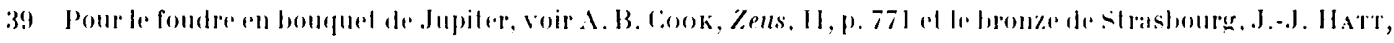

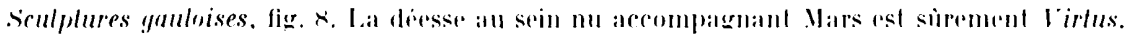

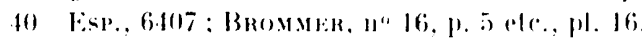

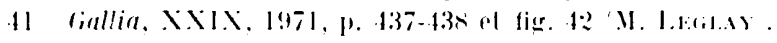


malgré son peu dimportance numérique. confirme done l'existence d'un lien entre la série Cil et la serie 11 ; de la premiere elle conserve la forme grenerale, landis que son ironographie est identique a relle de .11 .

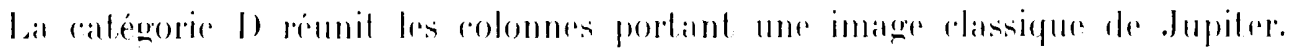

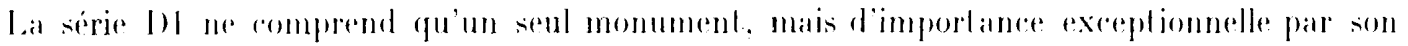
etal de conservation. la richesie de son décor, et le late qual soit date arec précision d'une époque fort ancienne par rapport à la plupart des colonmes de la sirie 11 : il s'agil de la colonne consacrée a

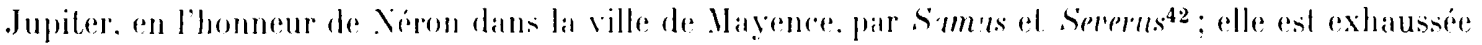
sur deux sorles parallépépépliques: l'inférieur porte les images de Jupiter el d'llereule. isolés el encadrés de pilistres baroques inspirés par le lVe slyle pomperien. sur les deux aulres faces sont groupeses deux divinites alfrontés selon un schéma romano-allique que nous retrouverons tout a

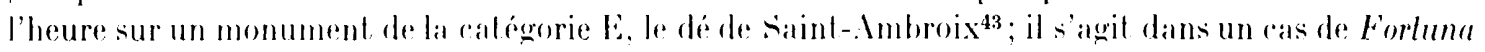
el de Minerve dans l'aulre de Mercure et d'une désse non directement identifiable; le second socle presente les loioscures et Ipollon (un has-relief est détruil). Iu-dessus d'une base altique, le fìt est découpe en ring lambours sculplés des images de dix-neuf divinites donl un bon nombre sont assez difficilement irlentifiables. Ie chapitean, corinthien normal. porte un de rechangulaire qui supportait la stalue de Jupiler en bronze. naturellement disparue. Ce qui nous imporle ici est de savoir si la colonne de Mapenere appartient a la famille que nous éludions. Xous répondons sans hésiler par l'affirmative. non seulement parre qu'il s'agit d'un monument dédié à .Jupiter, dont le

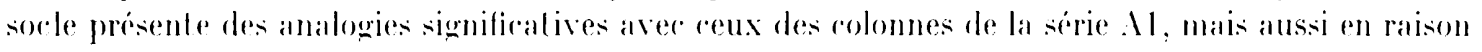
des concordances qui ne peurent ifte fortuiles aver des monumenls d'autres catrogries. comme le pilier de siant-Ambroix. Ge point alabli. quelle est la place de la colonne néronienne dans ul'arbre grenéalogiquen que nous essayons de dresser? Ce ne peul al re celle du prolotype non seulement a rause de l'antériorile du pilier des naules de Paris. mais pare que les diverses particularités des monuments de la séle 11 n'onl evidemmenl pas loules leur sourre dans la colonne mayencaise. Celle-ci. d'ailleurs. n'a pas déguivalent dans les autres parties de l'empire bien que la disposition des figures en frise sur les lambours rappelle les columnare eaplalae d'Asie Mineure! Il ne s'agil done pas d'un monumenl élranger implante en Ciaule. mais beaucoup plus probablement d'un monument gallo-romain. appartenant a un lype deja existanl dans la province. auguel ses anteurs onl voulu donner un aspect particulierement soigne et solennel.

Ja serie 1): est forme de colonnes dont le rhapitead supporte une image de Jupiler assis

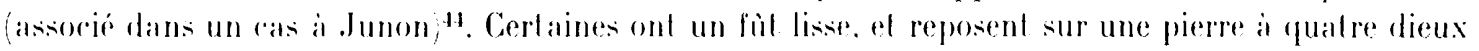
semblable à celle de la sirie 11 : elles forment le groupe l)2 a. Ce groupe ne paraîl en fait représenté

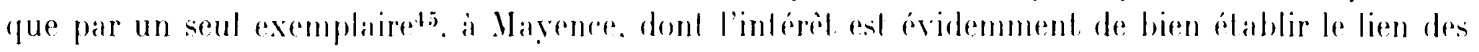

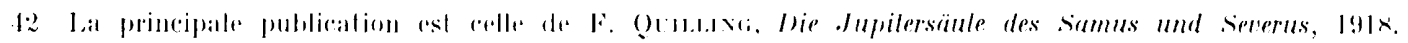

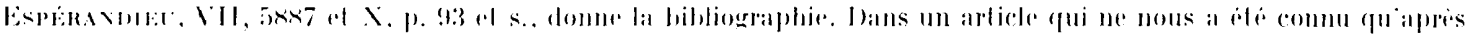

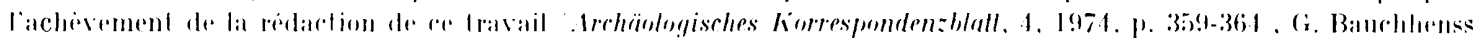

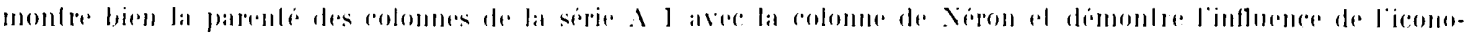

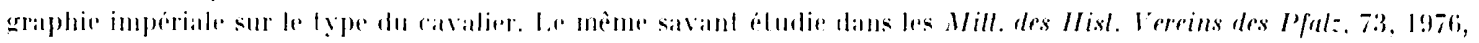

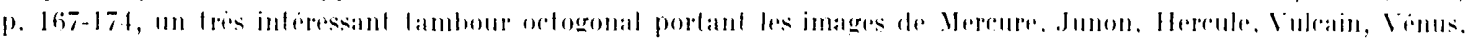

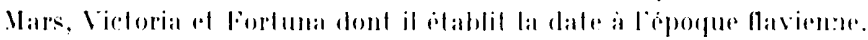

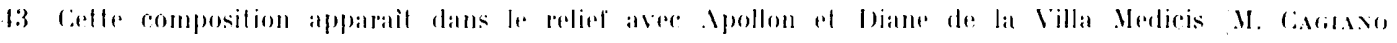

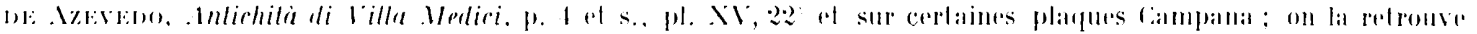

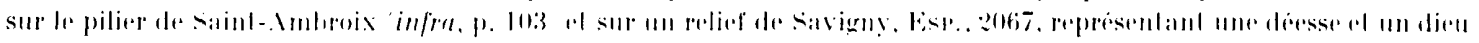

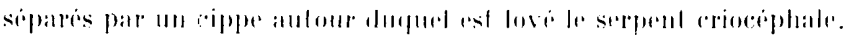

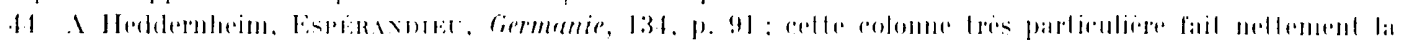

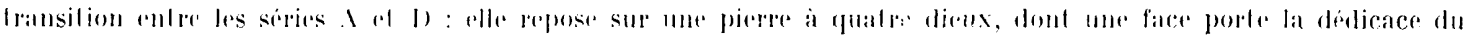

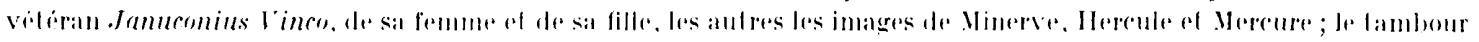

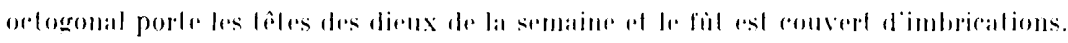

1.) 1 :SP. $7: 3: 7$ 
séries 11 et 1)2. Le groupe 1)2 b est represente par une douzane de monuments lous localisés en Germanie Inférieuret6. les fùls. géneralement recouverts d'écailles (mais il y a des exceptions). portent des images de divinilés superposées, laillés en demi-bosse a ce sont généralement Winerve. qui de beaucoup est la plus frequente. Junon el Mereure. Mais on voit apparailre aussi. a la place de ce dernier, Hercule. Apollon ol Vulain. Le choix des dieux est donc le mème que sur les pierres a qual re dieux. Quant au mode de présentation. son orique hellénistique est bien étable : on est parli de stalues accolées a la colonne el par lintermédiaire de statues portées sur consoles on esl passé a des images sculptées sur le fìl. en relief plus ou moins areuserti. Ces colonnes orneses se rencont rent a Cst ie a Tomi. en Afrique ou elles sont assez fréquentes. Il n'y a done pras lieu de postuler nécessairement, pour les colonnes de ciemanie une origine orientale imméliale.

lat serie 10:3 comprend un seul monument, de nature foul a fail particuliere; il sagil en effet d'une representation de colonne jovienne sur une mosaïque ; le serond lableau a partir de la droite de la rangée du haut de la mosä̈que-alendrier de Saint-Romain-en-fial Rhòne) représente une scene d'othrande a une divinite que (i. Lafaye avait idenlifie a Cérests sans que personne conleste depuis celle interprétation : nous avons pu montrer que cetle divinile est nue el quelle a pour allribul. d'une part une roue. d'autre part un foudre d'un type particulier. en fleur de lys. Il s'agil dome d'un Jupiler gaulois. S'il étail aceompagné d'un geanl ou d'un captif. nous pourrions le ranger dans notre série B ; mais iri le dieu es parfaitement parifique. comme reux de la série l) en général. La mosiäque de saint-Romain nous apprend done que l'on dressait sur des colonnes non seulement des images du Jupiler cavalier ou du Zeus classique, mais des effigies nues pourvues d'attributs reltiques : rertaines staluetles que nous possedons, comme relle du Chat elet so qui a les dimensions de l’image de saint-Romain, ont pù ètre présentées de la sorle la mosiäque de siant-Romain a en outre le tris grand intérît de nous informer sur le rulte qui était rendu aux colonnes joviennes : sur un autel paré de guirlandes el adossé au fùl. un homme en lunique, pieds nus, brùle de l'encens: une femme qui prie. la main droite levée, sest decouvert la poitrine, et tient une guirlande ; un vase au col long et étroit rontient sans doute le liquide d'une libation; les objets rultuels ont éte apportés dans une sorte de mallelle. Tous ces rites onl surement pour but d'obtenir une bonne récolte. Jupiter, dans le Vidi de la (jaule, recoil l'épiihète de frugifer, réservée en Afrique à salurne ou à Plutonj. Le probleme est de savoir si le mème culte était cébébé devant les colonnes de la série 11 ; des aulels ont été lrouvés associés a plusieurs d'entre elles : d'autre part. P. Lambrechts a conclu du fait que chez les I ingons. les colonnes ont ete souvent trouves dans des fontaines. que le dieu au rheval était un maître des eaux souterranes.52. Celle interprétation. qui se heurte a une foule d'objections 53 . ne reçoil pas de confirmation de la mosaïgue de saint-Romain. car aurume soure n'est apparente dans le lablean. I. culte du Jupiter gatulois a donc surement un aspect agraire, qui d'ailleurs nen épuise pas foules les virtualites. Volons enfin que nolre mosäque alleste la pratique. dans la vallée du Rhòne ou les colonnes du lype 11 sont rares. d'une forme cultuelle. étroitement apparentée.

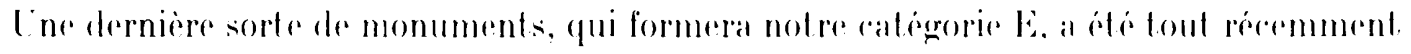

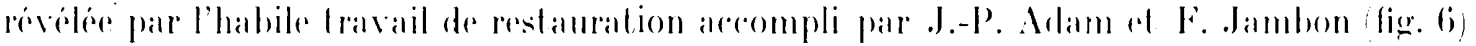

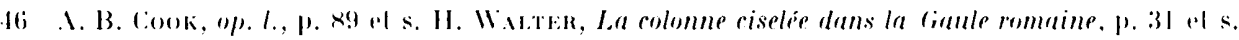

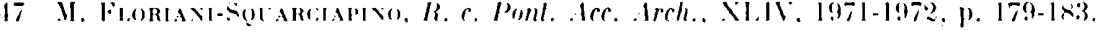

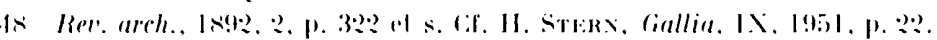

19 Bull. Sinc. Nat. des . Inliquaires de lirance, 197.1, 1. 1:7-1:37.

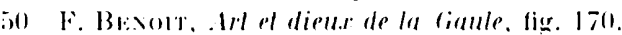

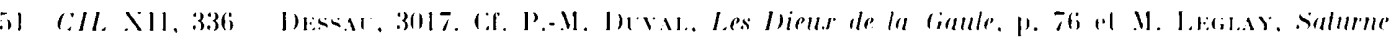
1fricuin, 1. 1:3.3.

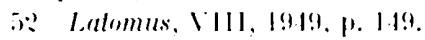

5.3 C.1. J. ne VRms, op. I., J. 11. 


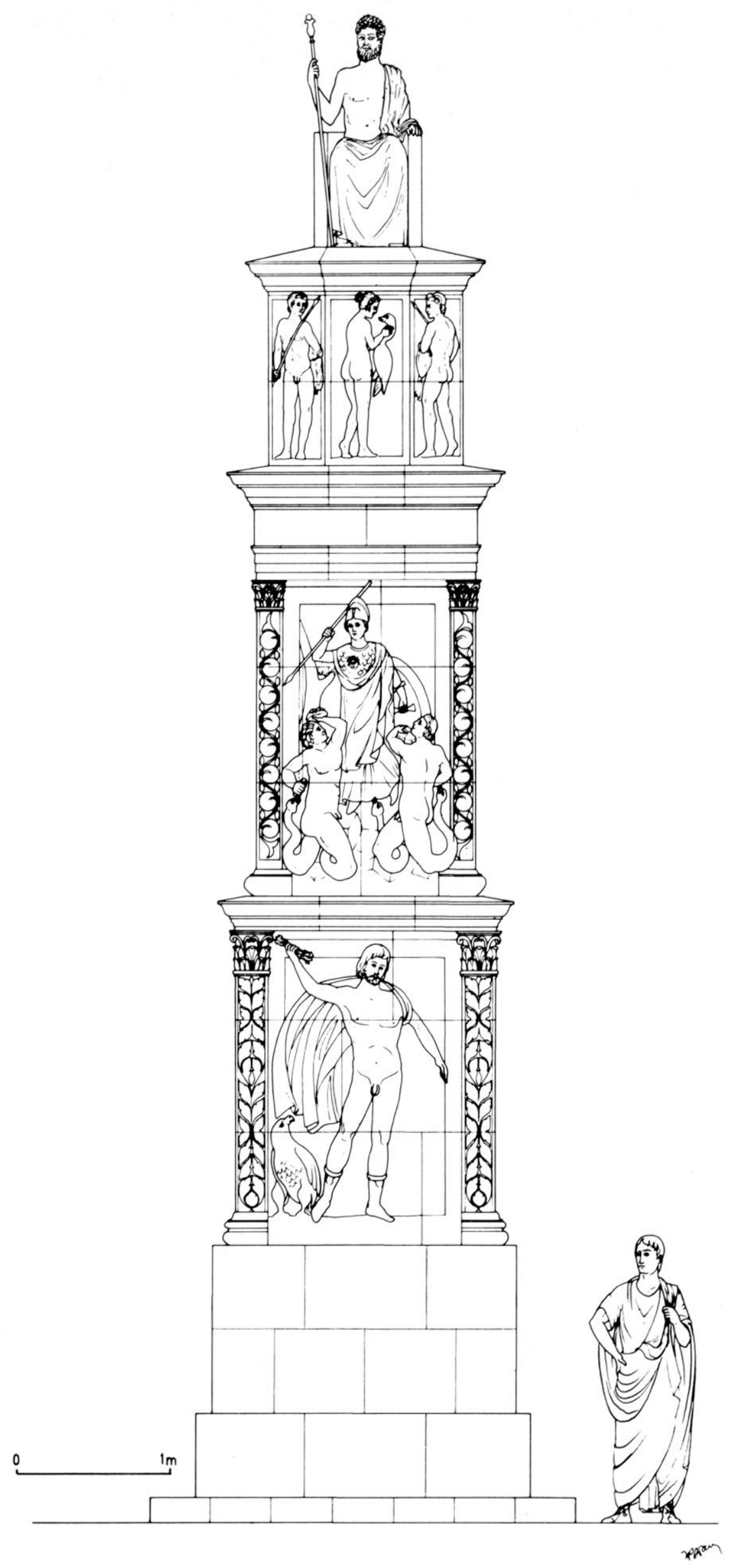

6 Reslilution du monument d'Yzeures par J.-P'. Alam. 


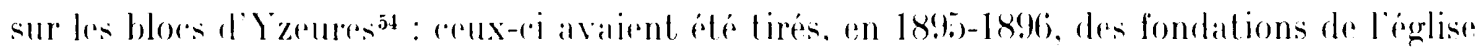
où ils araient ete incorpores en vertu d'une pratique dont les monuments joviens de Ciaule. ont fail maintes fois les frais ${ }^{55}$. Ils adalent devenus, depuis. soure diembarras pour les

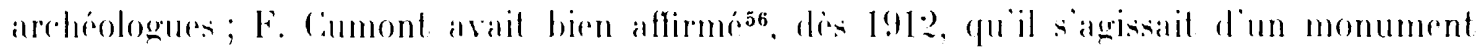

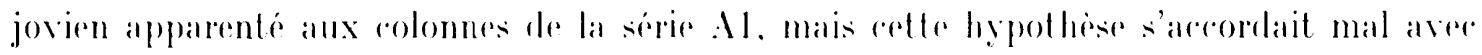

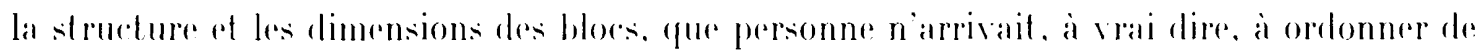
faceon sal isfaisante. I.-P'. Adam a résolu le problime arehitectural en montrant que les

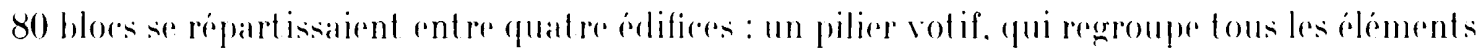

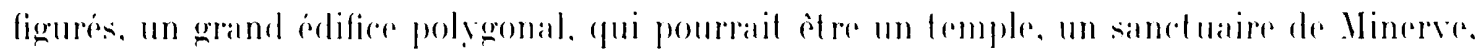

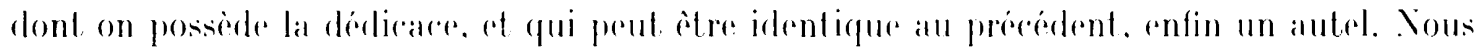
sommes done en presenence d'un sinctuaire important, dont te pilier votif ne serait quion

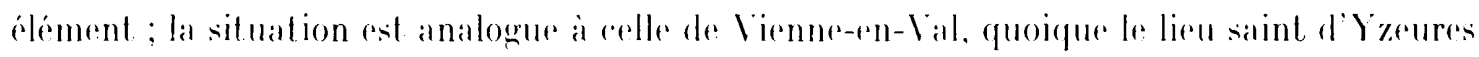
apparaisse moins important et beaucoup plus homogene chronologiguement, tout fe travail ayant ate semble-t-il. areompli. en deux generations, aux frais l'une famille de notables

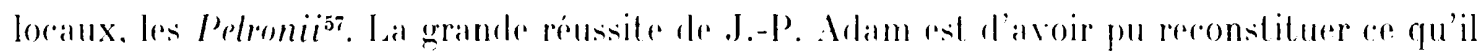
appelle le pilier votif, formé par la superposition d'un socle en grand appareil non décoré. d'un premier étage quadrangulaire orne sur ses quat re faces des images de Jupiter fulgurant. Apollon, Vars et Vuleain a somme une gigantesque pierere à quatere dieux); d'un second datage egalement quadrangulaire, mont rant Vars et Vinerve comballant les géants, Hercule délivant Hesione. et Persére liberant Andromede ; d'un tambour octogonal avere les ligures de Léda, des llosecures, dre Xeplune, et d'un personnage dansiant. Sur la pyramide ainsi

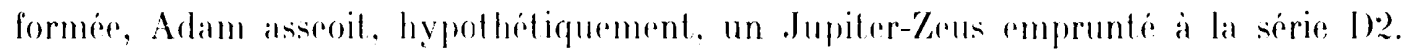

Cous voyons done apparaitre une nowvelle rategorie de monuments joviens, de

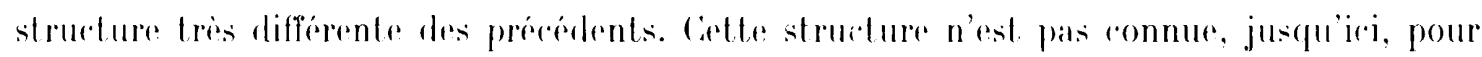
des monuments volifs; elle chail utilisie normalement pour des élifices funéraires, ou pour des monuments triomphaux, les trophées turriformess. En admetlant que la restitution d'Adam soil exalde. et nous ne voyons pas comment on pourail la contester. au moins

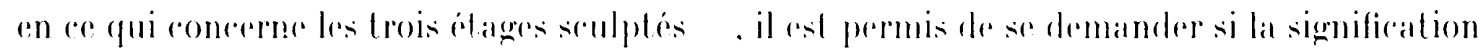

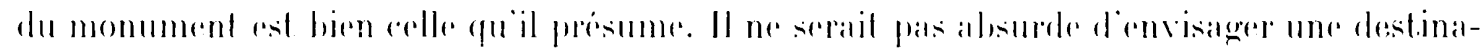

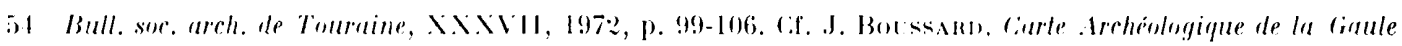

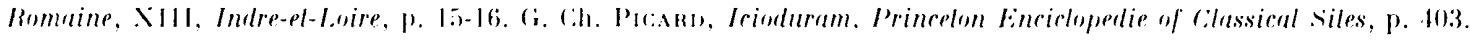

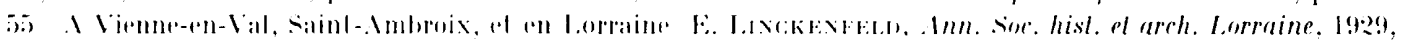
1). 1

56 Ker. arch., 1912, :, p. 210 ats.

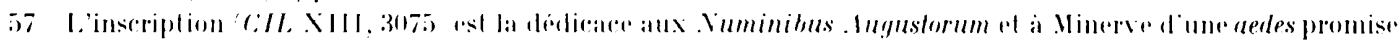

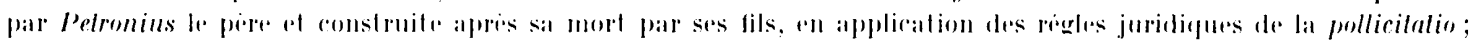

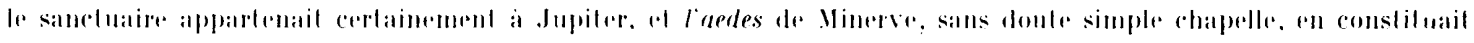

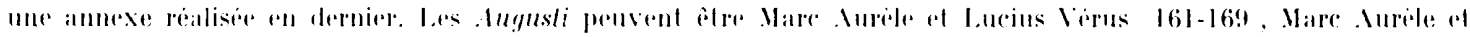

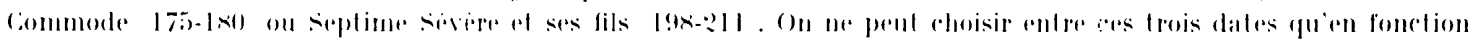

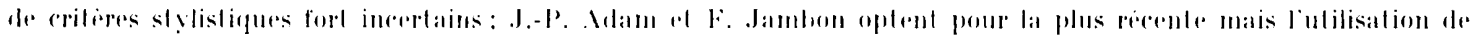

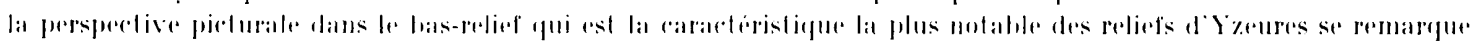

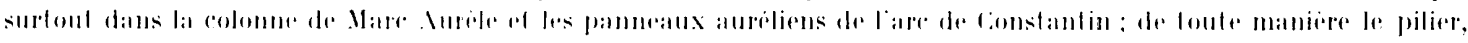

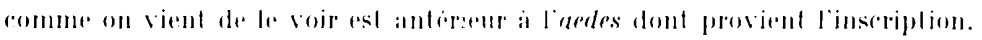

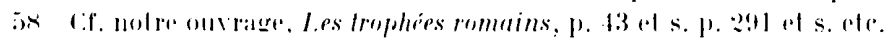




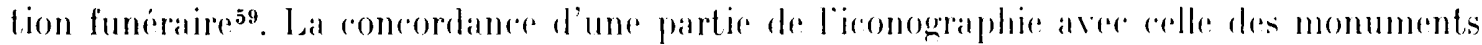

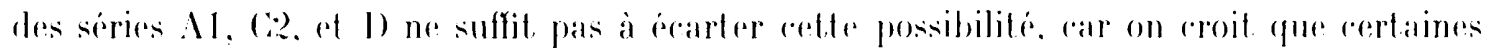
colonnes de la serie A1 alaient ete dressies all-dessus de tombes ; la presenere a lienne-entVal, d'une statue de fauve devorant, a prermis de confirmer l'hypothese de relations entre

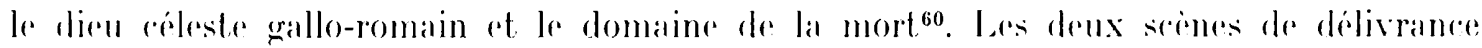
d'hérönes scupteres an second etage de la tour d'yeures appartirnnent au répertoire funeraire normal de la Caule romainebi. La figure dansante de l’étage ortogonal. qua relle soit mâle ou femelle, appartient aussi à re mème prepertoire, et non à colui des piereses à quatre diewx ${ }^{62}$. Il serait tentant de voir dans le monument le mausolée de Petronius le pere, qui mourut avant davoir pu areomplir entierement la promesse faite à son payus, et dont les fils terminèrent la tàche. Cés arguments ne sont pass pourtant. décisifs. La délivranere d'Hesione a pour auleur llercule, fréquemment honoré sur les pierres a quatre dieux. méme an rlehors de la Germanie (en particulier à Vienne-en-lal ${ }^{63}$; la libération d'Andromiede glorifie indieretement Minerve, inspiral riee de Persée. Les deve cartons. d'origine sans doute.

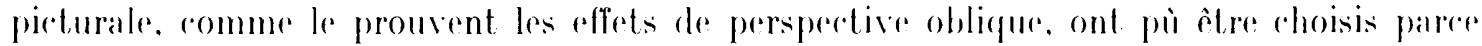
qu'ils présentaient les héroünes nues, er qui farcilitait laur assimilation à des nymphess locales ou a lénus : la presenee de Xeptune sur le tambour ortogonal indique sans doute qu'une sourer jaillissait dans la sanctuaire. Quant all danserur ou a la dansemse, il proul froguer l'un des amours de Jupiter.

Si nous passons du probleme de la forme à relui de la signification ideologigure. Ir pilier volif d'Yzeures presente des raratèeres qui le rapprochent des trophées. Nous avons montré

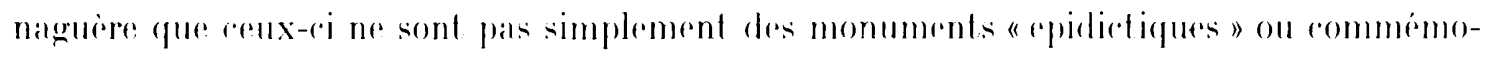
ratifs, mais de veritables sanctuaires, dédiés au dieu qui procura la vietoire; le trophée de

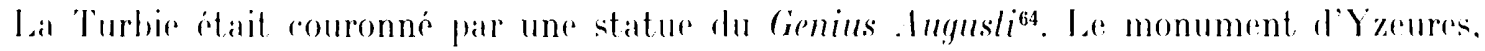

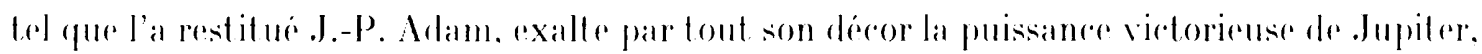

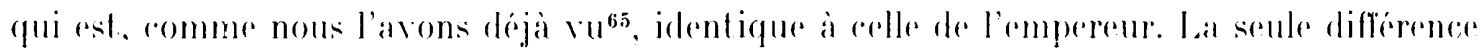

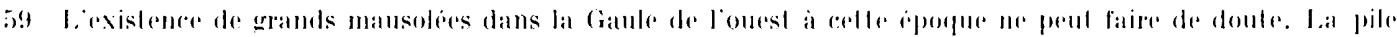

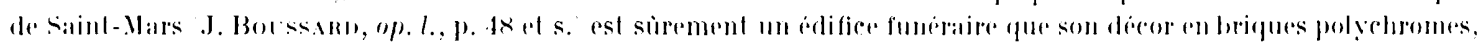

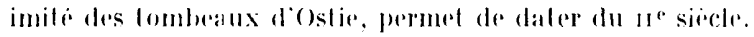

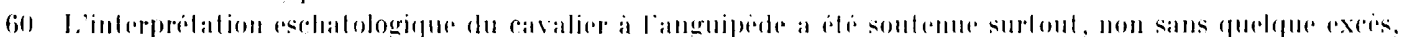

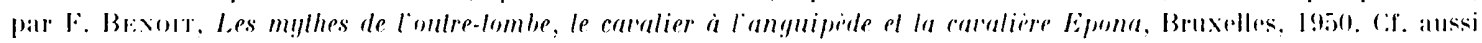

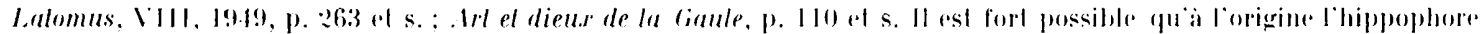

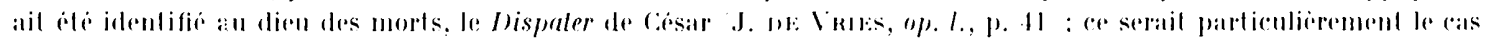

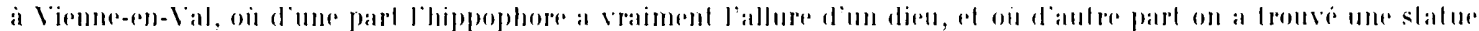

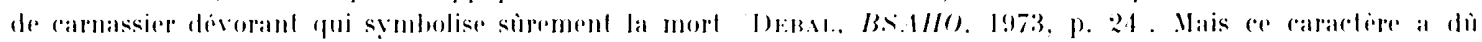

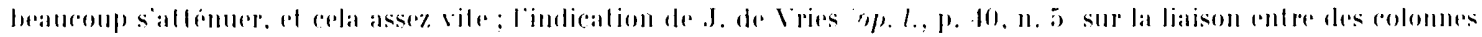

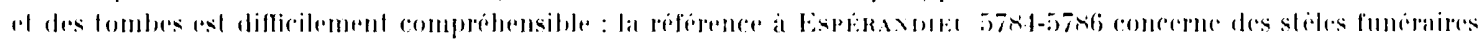

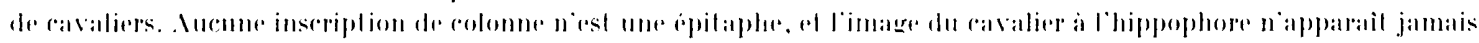

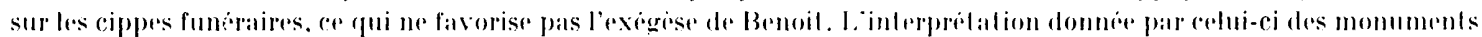

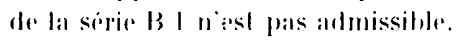

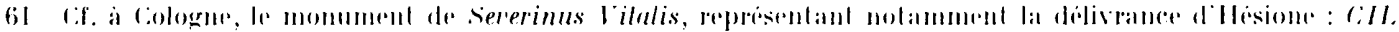

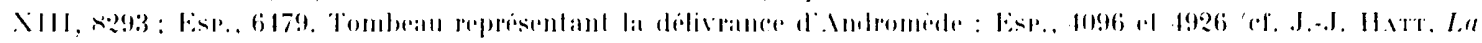
lomber yallo-romaine. 1. 195).

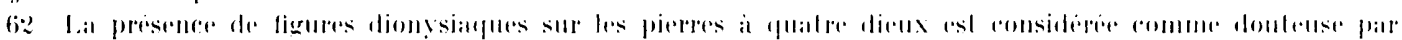
H:aug op. I. col. $31: 2$.

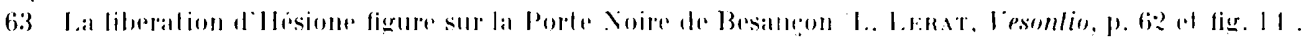

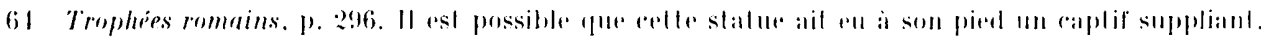

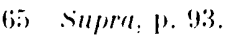




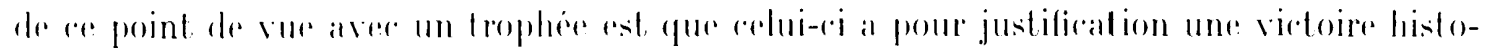

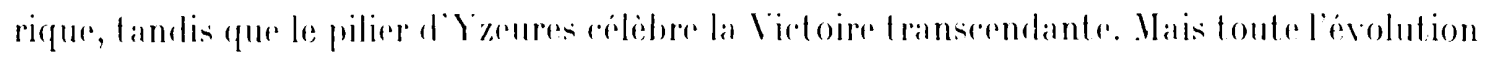
ideologicyue de l'Empire tend précisement a "transerendentaliser" la notion de Vietoirest. le monument s'explique done parfiatement en fonction de la pensée de son époquebr.

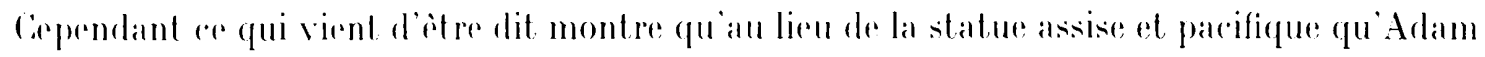
a placére en son sommet, il valudrait mieux installer un Jupiter combattant, soit carvalier.

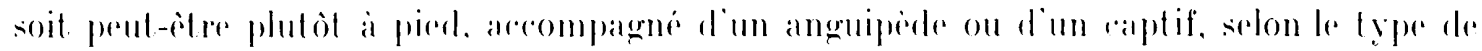
notere serie B.

Mais lexactitude de la restitution par Adam du monument d'Yzerures ne pourra etre

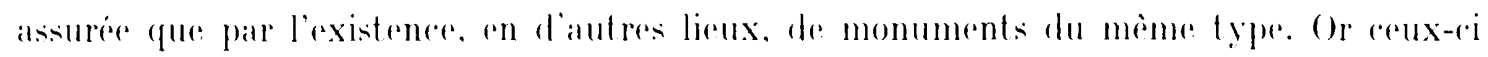
paraissent bien exister ; Arlam lui-mème avait rite le pilier de saint-Ambroix. an Musée de Bourges; nous pensons pouvoir ajouter deux aut res monuments. l'un d'Antigny, conserves a Poitiers, laatre de Brötzingene dans le pays de Barle.

Du pilier de saint-Ambroix ${ }^{68}$ subsiste un bloc quadrangulaire ayant pour seretion un carre de 1.15 m : fig. 7); (chaque face porte. entre deux pilastres en forte saillie, montés sur une base mouluré et décores de rinceaux, deux figures dont nous arons garde la partie inférieure; dans trois cass, il sagit d'une déesse drapere assorié à un dieu nu, selon le schéma romano-ittique que nous arous déjà rencontré sur la base de la colonne de Véron à Iayence 69. Deux des dieux sont aisément identifiables: Apollon, appuyé sur son trepied, at Vercure tenant sa bourse. Le troisieme couple est sépare par une sorte d'eminence ou de butte portant un petit animal qui pourrait etre un tres jeune ourson ; le dieu chausse des hottes de chasseur ; Ch. Picard avait pensé à I)ionysos, associè à Artemis ; mais Bacehus est peu populaire en Gaule. On songerait plus volontiers à Silvain représentant quelque dieu chasseur celtique; la déesse est sans doute Diane assimilée à Irlio, déesse reltique de l'ours ${ }^{70}$. La quatrième face montre deux jeunes garçons nus, les bras levés, sans doute des danseurs.

L.e site de Saint-Ambroix a rendu un tres grand nombre de reliefs gallo-romains, qui araient ete remployese comme ceux d'Yzeures et de Vienne-en-lal, dans les fondations diune ioglise mérovingienne. Ce sont pour la plupart des steles funeraires representant les defunts aver les attributs de leur condition sociale. Le grand pilier s'en distingue immédiatement; cest le seul qui porte des images de dieux; ceux de ces dieux qui peuvent ètre identifiés n’interviennent jamais dans l'iconographe funéraire gallo-romaine; il s'agit done d'un monument votif (figr. 8). Vercure et Apollon sont très fréquemment représentés sur

66) Trophées romains, p. 119 1.1 s.

67 Loxistence des momuments du lype d'Yeures, appapentes aux I rophees par la signification et par la forme, mais dont l’illustration est exclusivement mythologique, explique pent-être le singulier emploi de la mỵthologie dans

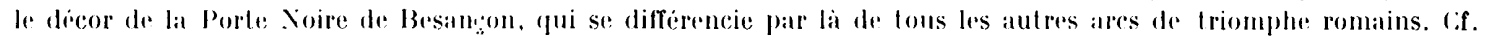
I. I,:RAт, op. l., p. 62. I.a gigantomachie est sculpté a l'archivolte.

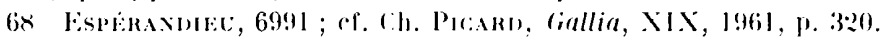

69 Supra, n. 13.

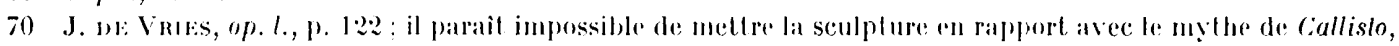
mais il pourrait s'ageir d'ume histoire amalogue: l'ourson serait-il le fruil des amours du dien et de ba deesse ? sur

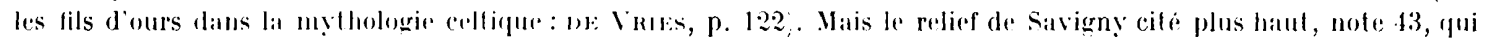
represente un dien el me desse sejares par un cippe aulour duquel est love le serpent criocephale, invite a imaginer d'autres types de rapports antre le conple divin of l'animal surnalurel. 


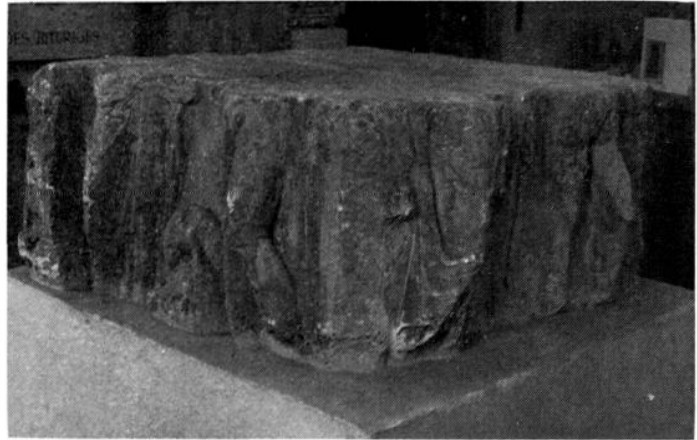

7 Pilier de sainl-. Imbrois.

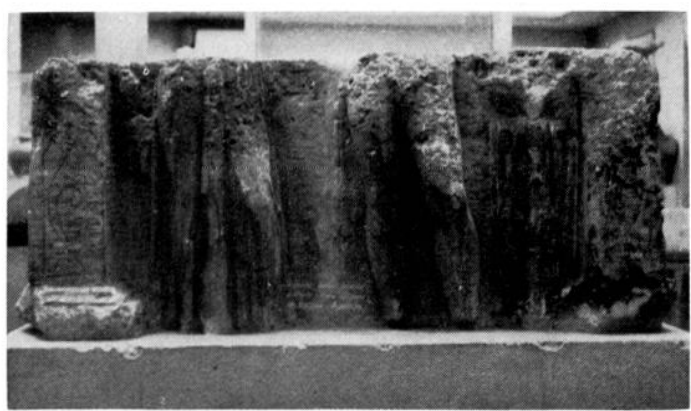

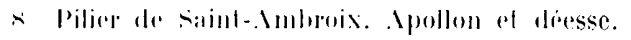

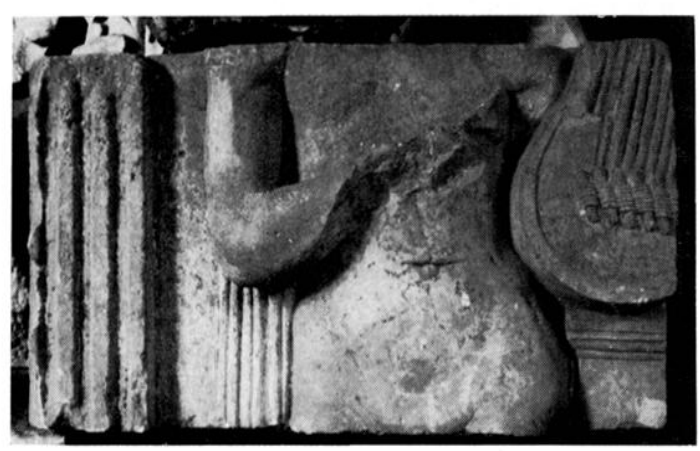

9 Mirliel dintign all Vusine Me Poitiers.

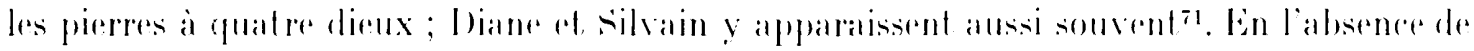

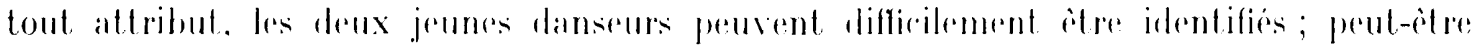

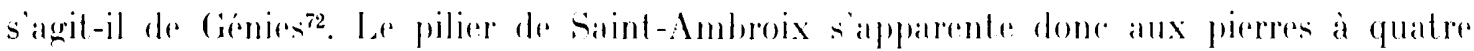

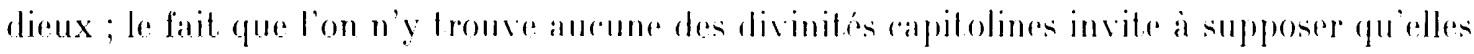

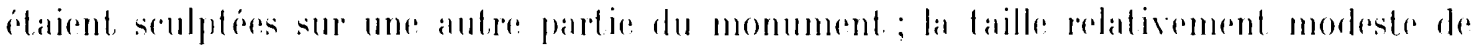

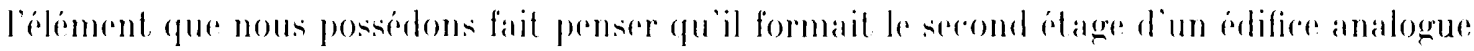

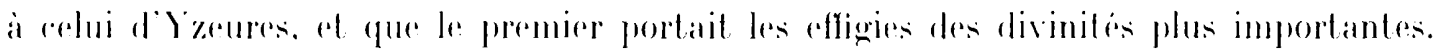

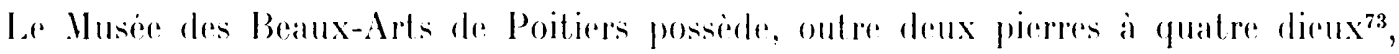
l'angle d'un bloc sculpte découvert à Antigny pres de. Iontmorillona fig. 9j. I'n pilastre cannele marque la jonction des deux falees, conserveres. lane sur une longueur de $0.71 \mathrm{~m}$, lautre sur 0.45 $\mathrm{m}$; la hauteur artuelle du fragment est de 0.10 m. Sur la fare la mieux

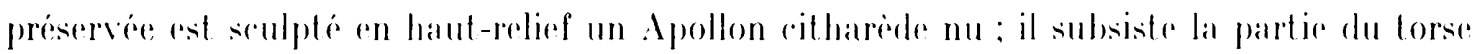

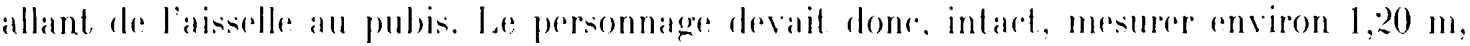
romme reux du second atage d'Yzeures ; sur la fare rontigue se voit le reste d'un dieu nu portant une haste Mars, d'apres Esperandieut). La position des personnages tout rontre

7 K. HAvi, op. l., col. 311-31:2.

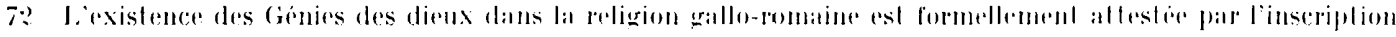

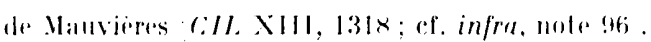

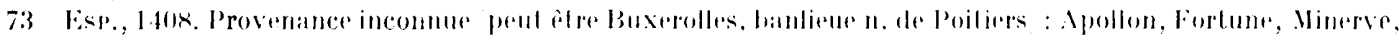

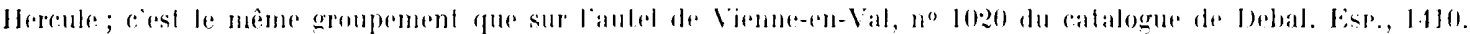

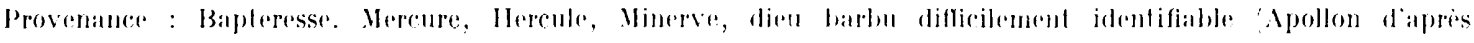

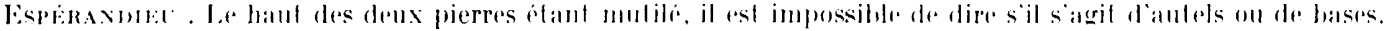

71 Est.. 111:. 
le pilastre d'angle donne à penser qu'ils etaient deux sur chaque face. Pour les raisons indiquées plus haut. à propos de saint-Ambroix, il parait certain que te monument etait rotif et non funeraire. Ie travail à la fois sophistique ot maladroit est très différent de celui d'Yzeures.

Certains monuments classés parmi les pierres à quatre dieux mais qui se distinguent par leurs fortes dimensions on leur ornementation originale comme les piliers du Pontau-Change et de saint-Landry à l'aris ${ }^{75}$ pourraient appartenir à la catégorie que nous étudions; c'est par exemple le cas d'un "autel " de Vayence portant sur chaque face un couple de divinités fort proche de ceux de Saint-Ambroix ${ }^{76}$ : Hercure et une déesse, Apollon, dont la compagne, assise et remi-nue ressemble à une nymphe, Diane et le dieu au maillet. enfin un Génie et une Abondance. La pierre de Brötzingen, dans le pays de Barle\#, doit aussi ètre prise en considération, non seulement à cause de ses dimensions relativement fortes (section de $0,82 \mathrm{~m}$ de côté) mais surtout à cause du caractere aberrant, par rapport au répertoire ordinaire des l'iergöllersteine, de son ornementation : elle présente léela. Hélios, Vulcain, et une déesse drapée, inirlentifiable. Labsence des dieux capitolins, de Mercure et d'llercule, peut s'expliquer seulement s'ils étaient figurés sur une autre partie du monument. c'est-à-dire sur un socle quadrangulaire de dimensions plus importantes placé sous le dé qui a été conservé ; nous sommes done ramenés encore une fois à une restitution analogue a celle du monument d'Yzeuresis.

Celui-ci appartient done à une famille plus répandue qu'il ne semble d'abord, unie par une étroite parenté idéologique avec la série Al ; nous pouvons dire que la série Al dérive d'un type de monument triomphal qui est la colonne portant l'image victorieuse, et la catégorie E d'un autre type de monument triomphal, le trophée turriforme.

\section{$* *$}

Le classement typologique auquel nous venons de proceder autorise, croyons-nous, un certain nombre de conclusions.

La première est l'affirmation de l'unite de toute la famille dont nous venons en quelque sorte de dresser l'arbre généalogique. Tous les monuments, fort divers, qui la composent sont unis par des liens, les uns idéologiques, les autres morphologiques, que nous avons essayé de mettre en lumière et qui sont assez étroits pour que nous ayons pu, à plusieurs reprises, expliquer une singularité d'un monument en nous référant à un autre, de catégorie,

75 P.-.I. Duvar, Puris anlique, p. 199-2013.

76 Esp., 5752

77 Esp., Germanie, 382

7o I.e relief de Bordeaux, Esp., 1106 , pourrait avoir fait partie de l'etage inferieur d'un monumenl du type d'Yzeures; l'exégese que nous en avions propose R.16; VIII, 1969, p. 208 et s. : Vulcain dressant un trophere, a che ecarté par Brommer, p. 1., sous pretexte que les attributs du dieu manquent; or si le marteau est brisie, son existence nest pas douteuse, car on me voit guere le personnage "boxant "de son poing le mannerfuin situé à sa gauche. Quanl au pilos, il a disparu avec le cràne mais la partie du fond qui na pas subi le striage impose a tout ce qui blait visible du monument s'éleve assez haut pour montrer que le personnage portait une coiffure. Iat sionificalion triomphale do la scene conviendrat parfaitement à un monument du type d'yeures. 


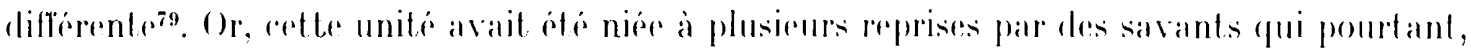
ont contribue très valablement. comme Haug, a faire progersser notre connaissaner de cette remarquable et singulière branche de l'art, romain.

La serende conclusion, déeoulant de la première, conererne la répartition géographique ret ehronologigue des monuments. Il avait semble normal de chereher loorigine du culte

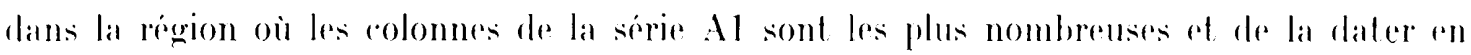
fonction des dédicaces portant mention d'une année consulaire. Or, sur cess deux points la statistique apparait trompeuse; si les monuments las plus nombreux se trourent de part de d'autres du Rhin moyen. ceux des vallées de la serine at de la looire apparaissent solvent plus anciens et plus riches de signification. Nous sommes, selon toute vraisemblanere en présence d'un phénomène que lhistoire religieuse contemporaine aide à romprendre : all xise siecte. la dévotion mariale, née autour de grands sanctuaires de pelerinagess s'est exprimée par une prolifération de monuments moyens ou petits grottes de Lourdes des jarlins de courents, statues de la Vierge de la sialette. ele.). qui s'est etendue à des régions

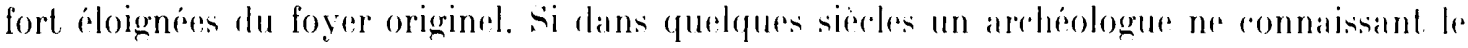
culte de lourdes que par res grotles artilicieHtes, essalyail d'en loraliser l'apparition a l'aide. d'une carte desdites grottes, il aurait toute chanee de se tromper. La mème crreur a ete certainement commise par cenx qui ont voulu expliquer le culte du Jupiter cavalier a partir des colonnes de cours de fermes ou de petits sanctuaires ruraux liés à une source ou à un cours d'eate. Il est bien établi que des colonnes se dressaient dans des villes, comme Partis. Strasbourge et Vayence et dans de grands sanctuaires, au Donon, a (irand. à Vienne-enVal, a liseolivese 81 est contraire a la logique de supposer que les monuments urbains copient ceux des campagnes, et qu'un grand temple emprunte une image a une chapelle

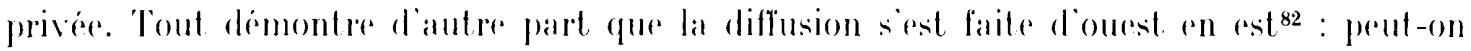

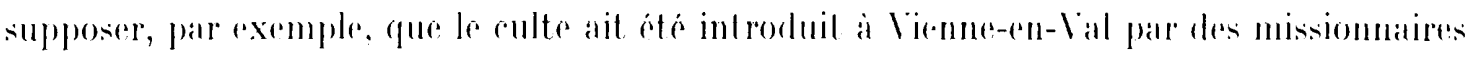
venus de Ciermanice? Ia dedicace d'une des pierres à quatere dieux a pour auteurs deux personnages, Perpeluus fils de liullus et Malermus fils de T'oulorix, qui représentent une

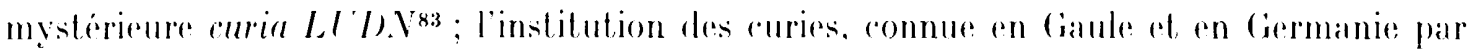

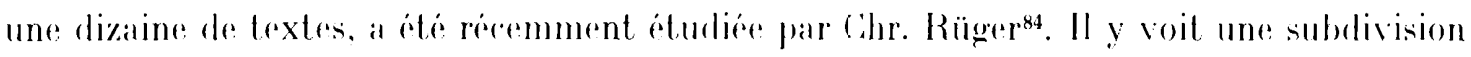
de la civilas à base csisentiellement ethnique, une sorte de clan à l'éensaise. Sans écarter rette possibilité, on peut en envisager d'autres: il pourrait s'agir par rexemple, d'assoriations qui se seraient constituées pour assurer la continuation du culte des dieux gaulois à la suite de la disparition du clergé druidique. En tout cass, Perpeluus at Malernus qui conservent. probablement sous le règne d'Antonin, la condition pérégrine, ne sont ni des immigrés ni

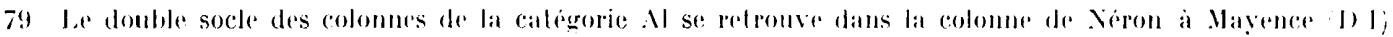

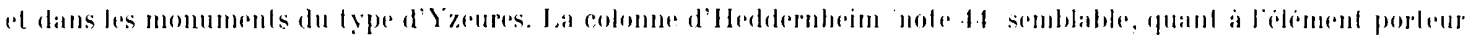

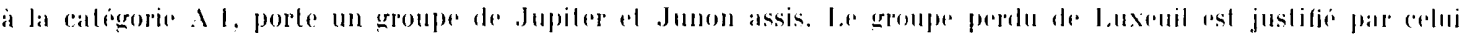
de Dompierre-les- Foglises, de la categorie B 1 et par l'equas lomitiani, ate.

80 J.-J. Hatr, strasboury an lemps des Romains, p. ix.

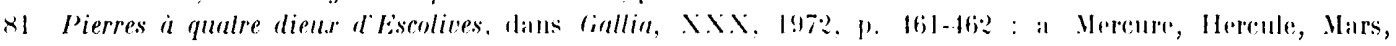
divinite feminine; b Hercule, Vimus, Vulanin.

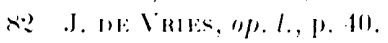

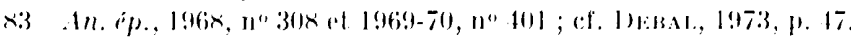

8. Epigraphische sludien, 9, 1972, p. 25]-260. 
Illustration non autorisée à la diffusion

10) Nonnaies des Andecturi. B.X. 67:21 ne 67:5).

des puissants: leur situation sociale rst inférieure a celle de la plupart des dédicants de colonnes en Germanie, qui sont souvent des décurions et quelquefois des soldats ${ }^{85}$; cependant r'étaient les principaux d'une communauté de droit public, puisque sal caisse est qualifiée de pecunia publica. Leur sanctuaire est le plus important qui soit connu chez les Carnutes ${ }^{86}$, et remonte probablement à une date assez haute ${ }^{87}$.

De tels faits permettent de supposer que le culte est né en celtique et qu'il a été importé en Rhénanie par des Cianlois mobilisés dans l'armée ou attirés par la prospérité du pays frontiere ${ }^{88}$; de mème le .Horcure du Puy-de-l)òme a été amené sur les bords du Rhin par des Arvernes immigrés qui lui ont ronserve son epithète ethnique pour bien te distinguer des Mercures indigenes ou de cenx apportés par d'autres Gaulois : l'appellation Arerernus donnée à Mercure est altestée sept fois en Rhénanie et ne se rencontre que là ${ }^{89}$.

L'hypothèse deviendra une quasi certitude si nous examinons l'origine de la représentation du Jupiter cavalier qui, si elle n'est pas l'unique type iconographique du Jupiter gallo-romain, est le seul tout à fait original et propre à cette partie de l'Empire ${ }^{90}$. Yous avons deux très fortes raisons de penser que les Gaulois indépendants de La Tène III représentaient certains de leurs dieux en cavaliers:

$1^{0}$ La numismatique des derniers temps de l’indépendance reproduit, comme l'a prouvé P.-.II. Duval, des images cultuelles ${ }^{91}$. Certaines de celles-ci sont équestres; or si les chevaux des monnaies gauloises tirent leur origine de thèmes ampruntes ces thèmes ont été rapidement nationalisés of ont servi à exprimer des idées proprement celtiques ${ }^{92}$. Cé sont surtout les monnaies armoricaines qui présentent des cavaliers ou des auriges guidant des chevaux androcéphales accompagnés de roues plus tard symboles de Jupiter - ou de

Xi) K. Hat:, I. l., col. 315.

86 DrвaL, 1973, р. 46-47.

87 les différences stylistiques entre les monumbuls indiquent une longue ulilisation du sanctuaire; les documents les plus anciens sont sans doute la slatue de cavalier, qui, comme nous l'avons vu, se situe parmi les plus archaïques, et pourrait, du fait de sa ressemblance avec la slede de C. Romanius : Esp., 5xise remonter au milieu du

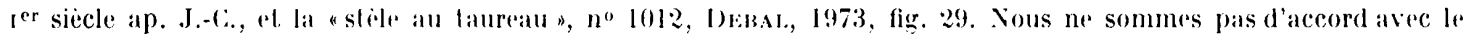
commentaire que bebal dome de ce document que rien a notre sens ne permet de rallacher au culle de (aybele.

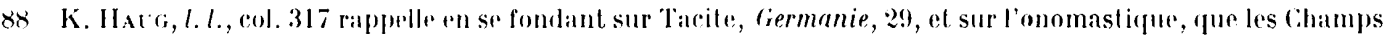
Decumales itaient penples de colons ganlois.

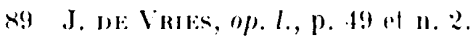

90 Les autres images de Jupiler cellique ne se singularisent que par des all ributs : roue, spirales evoquant l'aclair, foudre en fleur-de-lys. Tres rares sonl celles, qui, comme le petit bronze de Strastourg, J.-J. Hatr, Sculplures

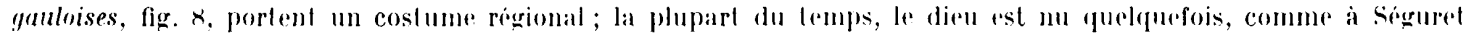
Vaucluse, F. Bexort, Arl el Dienr, fig. 16x al a Vaison, il porte une cuirasse de type impérial.

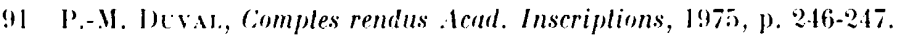

92 (A. J. Buyer, Wélanges arch. el hist. École française de Rome, 62,1970 , p. 15-41. 
lyres - plus tard symboles d'Apollon ${ }^{93}$. Particulièrement intéressante est une monnaie des Andecavi (fig. 10) présentant au revers "un rheval androcéphale à droite; au-dessous le. buste d'un homme, bras étendus, tenant de ses mains les pattes du cheval "94. Ce personnage. à mi-corps, soutenant les sabots du cheval divin que guide un aurige est évidemment. l'ancêtre des hippophores d'époque impériale qui soutiennent eux aussi les sabots du cheval jovien, de leurs mains ou à l'aide de béquilles.

$2^{0}$ Le terme Alepomarus, qu'on s'accorde à traduire « le grand cavalier " ${ }^{95}$. sert d'anthroponyme mais qualifie aussi des divinités. Ionné à Apollon par une inscription depuis longtemps connue de .lauvières dans l'Indre ${ }^{96}$, il vient d'apparaitre associé à Vercure dans une dédicace de Rennes ${ }^{97}$. J. Bousquet, qui a publié ce texte, pense à un dieu caralier gaulois, dont les images, dit-il, seraient difficiles à trouver, et qui aurait été assimilé tantôt à Apollon, tantôt à Ilercure. Cette théorie ne nous paraìt pas satisfaisante pour plusieurs raisons ; il serait surprenant qu'd tepomarus ait été en quelque sorte monnayé en deux dieux qui, s'ils sont souvent associés, restent bien distincts, et qui n'ont. dans l'ironographie: gallo-romaine, aucun rapport avec le chesal ; cet animal, faut-il le rappeler, etant attribue à Jupiter, à Epona, et, moins nettement, à Vars ${ }^{98}$. La fonction calvalière nous paraît, d'autre part trop temporaire, et même accidentelle, pour suffire à définir un dieu; enfin, il est toujours gênant d'arlmettre qu'un même vocable ait servi à nommer un dieu et un homme On échappe à toutes ces difficultés en considérant Alepomarus non comme un nom, mais comme une épithète ; les Gaulois de La Tène, dominés par une aristocratie de "chevaliers". devaient tout naturellement imaginer leurs dieux chevauchant, de mème que les combattants espagnols de la reconquisla n'hésiteront pas à jucher sur un destrier saint Jaceques,

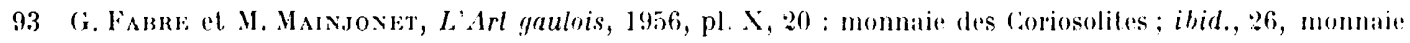
des Abrincalui; chevaux androcephales accompagnes de lyres. Pl. $\mathrm{X}, 11$, Veneles : an revers, cheval androcephale; derrière lui, une roue; sous le cheval, génie aile.

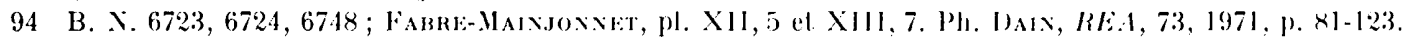
Ph. Dain demontre que ces monnaies, dont la frappe s'echelonne sur toute la premieme moilie du ler s. av. J.-li., onl eté émises par les Namnetes, et non comme on le croyait jusque-la, par les Andecaves. Il reconniât bien dans le type du revers, un génie hippophore. (or, les hippophores ne sont representes dans l'art antique que par les trois acrotipes

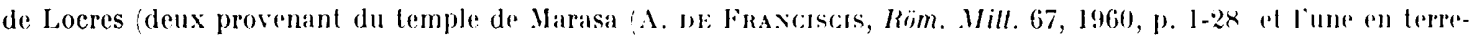
cuite, représentant un cavalier supporte par une sphinge el provenant du temple de Marafioti A. 1r: likaxciscas, Locri anlica, fig. p. 15. Ce dernier groupe est etrangement analogur: à des plarques frabriquées en Alsace par le faussaire Kaufmann, et qui s'inspiren! peutêtre d'un original aulhentique 'Lsp., 5473 at 5891 . Le type de lhippophore est trop extraordinaire pour quion puisse supposer quil a cte imaginé independamment trois fois dans le monde antique, en particulier par les Celles dont la capacite a creer des themes iconographicues semble avoir éte tres faible. Il faul

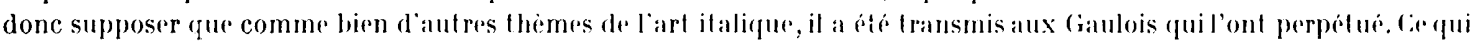
ne signifie nullement - - la se trouse la fatibesse du raisomement de k. Benoit - - qu il ait conserve une valeur identique tout au cours de son histoire. La signification eschatologique des groupes de locres-Marasa défendue par

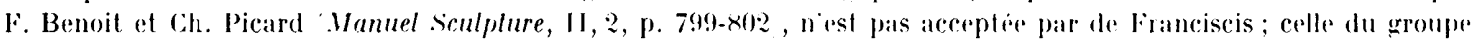
de Marafioti parait difficilement contestable, le caractire infernal de la sphinge datant admis par toules les civilisations antiques.

95 Le Rotx, Ogam, XI, 1959, p. 219.

96 CIL XIII, $1318=$ Dessac, 4637 : . Yum. Aug el Gienio Apollinis/.Mlepomari Iul. Alrieclus Cirasanti fil. el Iul./ Gnaius Atrecli fil. d. s. d.

97 J. Bousquet, Gallia, XXIX, 1971, p. 109-117.

98 Les ingénieuses spéculations de nombreux savants par exemple: F. Tuwwoxt, Divinilés el sancluaires, p. 47 et s.) se heurtent, au fait que l'art antique ne nous a légué aucune image de Mars cavalier; aussi est-il bien improbable que le débris de cheval harnaché n 1010 de Vienne-en-Val ait appartenu à une slatue équestre de . Vars, comme l'envisage Debal : $35.1 / 10,1973$, p. 20 : 
pourtant bien mal prepare a ref exereiere! Ceux que les Romains nommeront .Jupiler. Apollon. Nereure et sans doute aussi Vars etaient ainsi representes. I) antre part. la monnaie des Andecari montre que le vieux schème hellénique de l'hippophore arait déjà été adopté. sans doute pour illustrer un mythe de lutte entre le dieu du ciel et celui des enfers ; dans ce

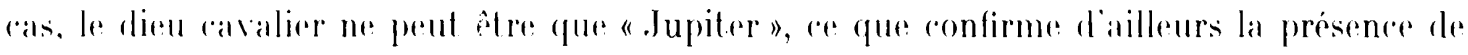
la rolle sur d'antresi monnaies.

La ronguete va rentrainer de nomvatux developpements; d'abord les images d'Apollon

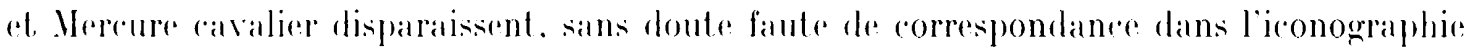
rassigue. Au rontraire le eroupe du Jupiter aralier et de l'hippophore bénéficie de sa ressemblance aver des groupes triomphamx romains representant un vainqueur à cheval trer-

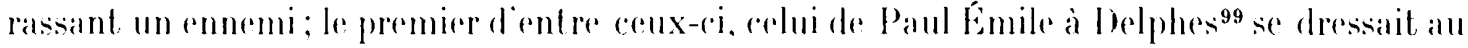

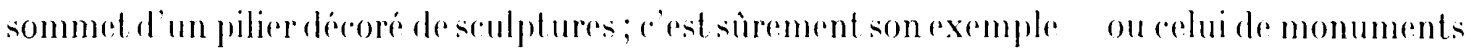
analoguess qui a conduit les artistes gallo-romanins à plarer des groupes du cavalier céleste all sommet, de piliers ou de colonnes décories. Gathe fusion d'un thrme iconographique

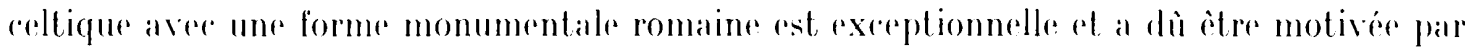

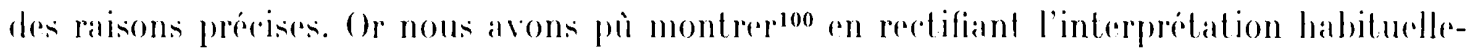

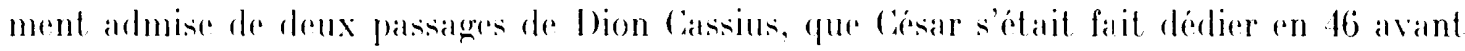
J.-C... alu Capitole, un groupe qui le représentait an rhar, s'alevant all-dessus d'une image courhee de l'oikoumene. Il existe une singuliere analogie entre ce monument et les groupes a l'hippophore; césar. qui favorise a cette époque le developpement du culte de Jupiter Julius, se presente. sinon romme une hypostase, du moins romme un associo du dien suprême; l'oikoumene est, la terre habitée au sens grographique, Landis que l'hippophore gaulois, màle ou femelle, représente au sens métaphysique et de manière imprécise l'ensemble des forees rhtoniennes. Chareune des deux notions opposéses dans l'une ot lautre des calegories monumentales, est ainsi analogue, sinon identique. Aussi n'est-ce pas faire une hypothese invraisemblable que de supposer l'existence, dans une ville on un sanctuaire de Gaule, d’un pilier couronné d'un groupe où le cavalier doté à la fois de l'apparenere de césar et des attributs joviens terrassait un monstere infernal, et dont le fìt portait l'image des dieux mobilises an service do l'imperalor.

Apres la mort de César, Auguste et surtout Tibere no s'engagerent quaver prudence at. mème répugnanese sur les voles conduisant à l'apotheose ; rependant la formule imaginése par lesiar ou a son profit ne disparut pas, paree quielle se pretait parfaitement au syneritisme antre la religion celtique et la romaine, qui etait une des préoceupations du gourernement impérial, désireux d'éliminer definitivement. le druidisme. Le pilier des nautes, dont la rédicace proure qu'il elait rouronné d'une statuo jovienne et lasses planer un doute peut-ètre voulu. sur les rapports entre le dieu et l'empereur ${ }^{101}$. apparait lout à fait caractéristique de centle éperpue de transition.

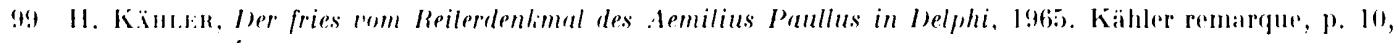

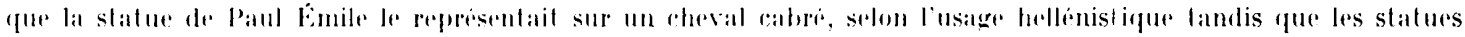
iofuestres depoque impreriale representent le cheval all pas. I.es statues du Jupiter gallo-romain se ratlachent ividemment a la lradilion hellonisliqute.

114) Ret. arch.. 1973, 2. 1. 361 +. s. .

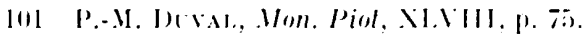


C'est à la génération suivante que notre type monumental s'introduit dans la région rhénane, où il ra connaitre une fortune extraordinaire. Chose remarquable, cette implantation ne se fait pas à partir d'un seul prototype ; elle est réalisée à partir l'expériences multiples, qui se situent à des niveaux différents de la société. Lous voyons en eftet apparâtre dans la seconde moitié du premier siècle :

10 Des groupes du cavalier à I'hippophore, comme celui d'Ehrangio2, dont la ressemblance avec les stèles funéraires des cavaliers de l'armée du Rhin ne peut être fortuite et fournit un élément de datation. Dans ce cas, la référence à l'image impériale est abandonnée, ce qui est tout à fait remarquable ${ }^{103}$. C'es monuments sont l'expression d'un groupe sorial défini où les traditions celtiques sont encore fortes ; peut-être y a-t-il là une volonté d'alfirmation des auxiliaires originaires de l'intérieur de la Giaule, face à des militaires recrutés en Europe centrale ou en Orient, propagateurs de cultes d'origine asiatique. Ln tout cas, il faut noter la ressemblance entre te cavalier d'Ehrang et celui de Vienne-en-Val, ressemblance qui apparait surtout dans le harnachement du cheval, avec selle à pommeau et troussequin, fortement fixée par des sangles ornées de phalères aux jointures; des harnarthements semblables se retrouvent à Brives ${ }^{104}$, et à Cologne ${ }^{105}$; on n'en voit jamais dans les Champs Décumates; ils sont donc un indice de datation haute.

20 Des groupes manifestement inspirés par l'art aulique, comme celui de 'Tongres, la statue perdue de Luxeuil et celle de Neschers, où nous avons reconnu l'influence de l'Équas Domiliani.

$3^{0}$ Avec la colonne de Véron à Vayence, un type de monument encore plus marqué par l'art officiel ; il dérive sans doute des colonnes joviennes, assez nombreuses à Rome ${ }^{106}$, et sera probablement à l'origine des colonnes au Jupiter trônant de notre série l) 2. Én outre, il introduit le systeme du double piédestal et le type de composition à deux personnages que nous retrouverons dans la série E, à Saint-Ambroix.

$\mathrm{Au} \mathrm{II}^{\mathrm{e}}$ siècle et dans la première moitié du III $^{\mathrm{e}}$, l'évolution est fort différente dans les Germanies, y compris les Champs bécumates, et dans les Gaules. En pays rhénan, des deux

102 L'authenticité du "Bauernreiler" d'Ehrang Esp., 52.t6, avail elé suspecté par Hettner tet K. Haug dans loscaten, Viergöttersteine, col. 316 ; elle est definitivement domontroe par sa ressemblance avec le cavalier de Vienme-en-Val.

103 V. P. Nillsson (Archiv für Religionswissenschaft, XXII, 1925), p) 179, jugeait que le costume nuilitaire? avait fait penser à tort à une image imperiale; il est at tribue, ajoutail-il, à de nombreuses divinitres venérés dans les provinces, surtout par lintermedaire des soldats. Il n'y a pas de rapports directs entre le Jupiler gallo-romain

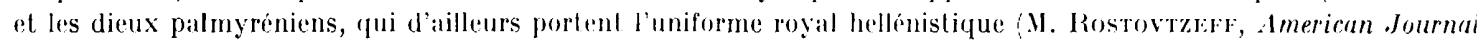

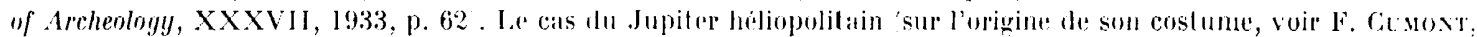

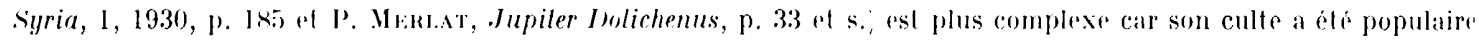
sur le limes germanique; il existe même une pierre à quatre dieux, provenant de Brötzingan Ess., Germanie, 365)

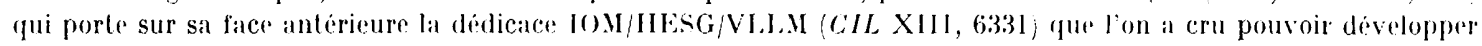
Iovi Optimo Maximo Ieliopolitano. Vais ce texte, en lui même énigmatiqur, ast surmonte d'un buste fort usé, nu el coiffe, semble-t-il, d'un bonnet phrygien. Il apparaît clairement en lout cas, lo (fue l'iconographie orientale n'a pu influencer la formation du type du Jupiter gallo-romain ; 20. 'qu'une des variantes de ce type a été créé dans les milieux d'auxiliaires gaulos de l'armee du Rhin, sous les regnes de claude ol de Xeron; 30 qu'une autre, influence par l'image impériale, et notamment par l'equas lomiliani, apparaît dans le dernier quart du der s.

104 Esp., 2752. S. Rexach, RS, IV, 340, 2. Provifnt du Cantal identique à : RS, II, 532, 4.

105 ESP., 6425. S. RENACH, RS, IV, 334, 4.

106 A. B. Cook, op. l., p. 45-47 d'apris Julius ()nswolexs, l8 el 19 et Dion Cassics, $37,9$. 
ròtés du fleure, on ronstate une standardisation qui aboutit à constituer définitivement la "série canonique" $\mathrm{Al}$; cette standardisation est liée à une prolifération qui multiplie les monuments dans les campagnes, sans d'ailleurs qu'ils diepraraissent des villes et des grands sanctuaires; produits en série pour des commanditaires aux ressources généralement modestes, cavaliers et pierres à quatre dieux accusent un appauvrissement dans le choix des motifs et dans l'exécution : les détails, comme ceux du harnachement, qui, à la période précédente etaient fignolés quelquefois aver maladresse mais avec minutie, disparaissent. 11 y a des exereptions, comme relle du pilier de Brötzingen ${ }^{107}$. déjà cité, et du monument des Slephanii a Ileddernheim ${ }^{108}$, date de 240 , un des plus tardifs par conséquent. Cet exemple prouve que la dégénéreseence n’est pas due à une déadenere artistique mais aux causes économiques et sociales que nous venons d'évoquer; les Stephanii sont une famille de notables, peut-ìtre d'extraction servile; fe père est décurion de la civilas Taunensium; il a pu s'offrir un artiste relativement habile, dont la manière rappelle celle de la pierre à quatre dieux de la place Kléber à Strasbourog ${ }^{109}$. Stephanius a fait preuve d’imagination dans le: choix de l'ironographie aussi, en substituant aux images banales des dieux de la semaine crlle de divinités, Mars, Genius, Fortune, Cérès, Vénus, Victoire, qui appartiennent pour la plupart au repertoire des pierres à quatre dieux, mais qui recoivent ici les noms des enfants du dédicant, de doivent par conséquent représenter leurs Génies, les dieux correspondant aux garcons ot les déesses aux filles. Généralement le dédicataire se bornait à accepter l'iconographie que lui proposait te lapicide, si mème il ne choisissait pas dans l'atelier un ex-roto tout prêt.

Aussi ne faut-il sans doute pas attacher trop d'importance à la valeur religieuse de tel ou tel élément. La signification idéologique générale est claire : d'abord l'acceptation sans réserve de la religion politique officielle, manifeste dans la référence au culte capitolin ; lié à cela, une sorte de "stö̈cisme du pauvre", dont les articles essentiels sont la confiance dans la Providence, personnifiée, al ciel par Jupiter et sur terre par l'empereur, l'affirmation d'une virlus dynamique et militante, l'exégèse symboliste et moralisante de quelques mythes rassiques. La part des croyances indigenes est morleste : deférence polie à l'égard de Mercure, probablement considere comme le dieu gaulois far excellence, mais toujours maintenu dans un rang secondlaire, alors que le relief de Narbach lui donne cmphatiquement la première place 110 . Hercule est peut-être honoré, complémentairement, comme dieu de la Ciermanie, mais l'interpretation reste douteuse, car on le rencontre aussi souvent sur les pierres à quatre dieux de (iaule centrale et occidentale. I)iane et Silvain eux, sont bien res divinités topiques. mais tout à fait dépourvues de ces caractères aberrants qui dénoncent les dieux indigènes mal incorporés dans l'olympe.11.

En (iaule, les monuments sont moins nombreux, mais certains d'entre eux attestent par leur importance, la richesse de leur deror at son originalite, l'opulence du dédicant et sa

107 Siupra, p. 105 a nold 77

lox supra, p. (1) el note :25.

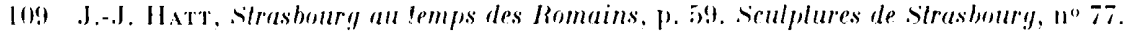

110 Esp. (iermanie, 6!5).

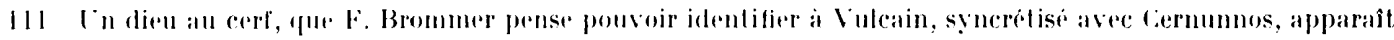

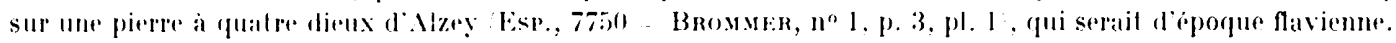


rulture. C"est d'abord le cas, bien entendu. des monuments du lype d'Yzeures : le serul fait qu'on leur ail donne une forme arehitecturale emprunté aux trophés prouve que cenx qui les avaient faits construire avaient médite sur cre que J. Gagé a heureusement nomme la "theologie de la Vietoire" Le choix des décors, représentant des mythes rares fleesse a l'ourson de saint-Ambroix) et celui des thèmes iconographiques suppose aussi une tres larope

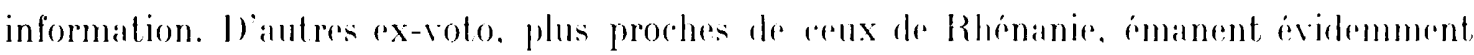
de fideles plus modestes, individus ou colled tivites commer la raria Ludn... de Vienne-en-Val.

Dans les deux serteurs, en tout ras. la grande vogue du culte correspond a la période

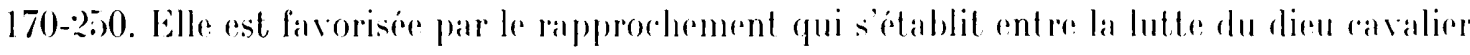

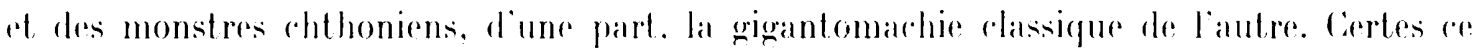

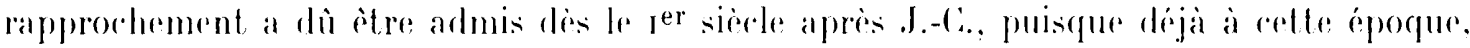
l'hippophore etait, saluf dans des ras exeeptionnels, romme colui du groupe de Vëenne-enVal, figure en anguiperde. Vais la gigantomardhe n'est utilisée de faren systématique au

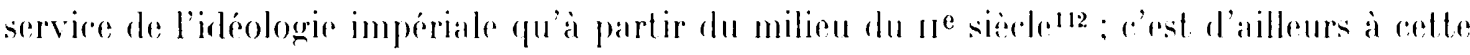
époque que les grandes frises pergaméniennes rederiennent a la mode al servent de modies alux artistes. I'antre part. le theme de l'empereur, a pied ou a cheval, foulant les ennemis. qui n’avait servi qu'exeeptionnellement aux Julio-Claudiens, reparaît prescaue sous lous les princes a partir de Domitien ${ }^{113}$. Sous avons deja parlé de ses contaminations constantes avec la representation du Jupita entique ${ }^{14}$. Cese formules brutales expriment bien le durrissement des Romains a legand des Barbares, surtout apres les invasions de 169, at le transfert de ce sentiment sur le plan métaphysique : de meme que l'empereur est de plus en plus assimile a la divine Providenere sesennemis sont identifies alux demons du mal : on ne silurait done: sectonner de voir de plus en plus fréquemment la Vietoire da lype de

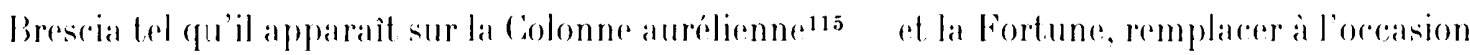

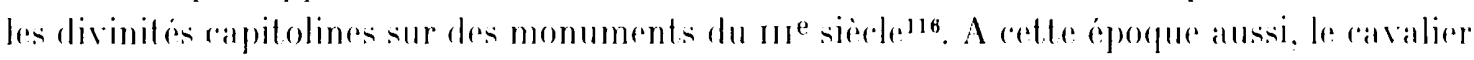
porte le plus sourent la tenure impériale, soit le grand uniforme aver cuirasse. soit plus sourent la tenue de voyage avere tunique al paludamentum. Ainsi l'interprétation historique du cavalier a l'anguipede comme symbole de la vietoire sur les barbares. valable en quelque sorte areessoirement lors de la rreation du theme, tend-elle a primer loutes les autres.

Les divers types de monuments joviens deviennent le mode favori d'expression du loyalisme dans les provineres gaulobises at germaniques ; on constate en offet que dans corte

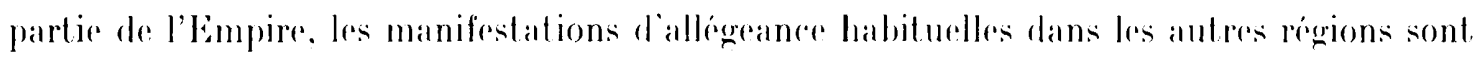

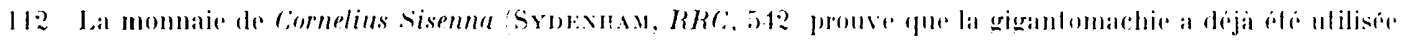

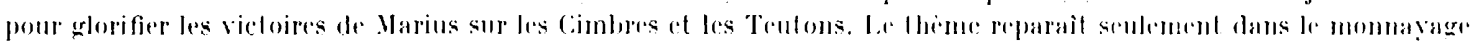

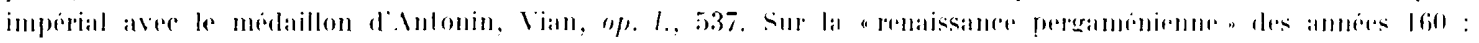

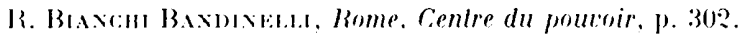

113 R. Bridiaxt, Gesture and ranli, p. $96-9 \mathrm{x}$.

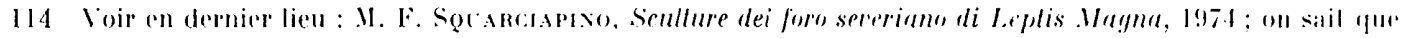

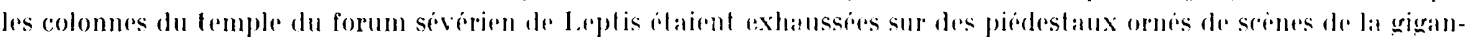

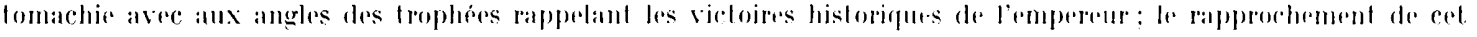

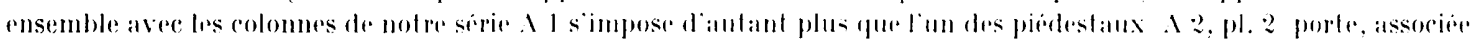

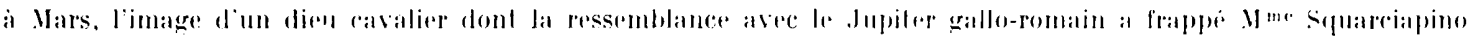

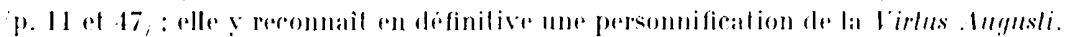

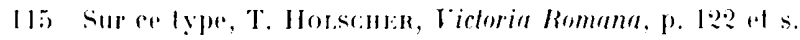

116 Esp. Germanie. 34!, 371 r. 
fort peu nombreuses : la rareté des portraits impériaux découverts en dehors de la Narbonnaise est par exemple tout à fait significative ${ }^{117}$. Il semble bien que les Gaulois aient estimé s'acquitter suffisamment de leurs devoirs à l'égard de l'Empire en dehors des rérémonies officielles célébrées au sanctuaire de Condale, ou, dans chaque cité, par les flamines, qui ont laissé somme toute peu de traces -... en associant la domus divina aux hommagres qu’ils rendaient à leurs dieux; la formule qui lui fait honneur, ou la dédicace all numen Augusli, précèdent presque toutes les inscriptions religieuses; mais, malgré la préséance ainsi affirmée du culte politique, la statue ou le temple, qui araient exigé l'essentiel de la dépense, appartenaient exclusivement au dieu. Les monuments joviens sont les seuls où la référence à l'idéologie officielle napparaisse pas comme une formule de politesse ou de préaution assez creuse. Ils représentent, en fait, la forme de culte impérial qui convenait all tempérament des Caulois, pare qu'elle s'accordait avec leurs traditions. Dans ce domaine, comme dans tous les autres politique et économique notamment..., les Gaulois ne refusaient pas d'appartenir à l'Empire, mais ils voulaient y participer à leur manière, en conservant, sinon une nationalité qu'ils ne pouvaient concevoir (du moins au sens que nous donnons à cette notion), leur personnalité et leur originalité.

\section{Gilbert Charles Picindo.}

117 Par exemple le tome IV du Recueil d'Espresaneser, qui courre te Bassin parisien, la Normandie ef la Bretagne ainsi qu'une partie de la Bourgogne, ne contirnt pas un seul portrait impérial dont l'authenticité soit insoupconnable.

N.B. Les clichés nous ont ite communiquies par .M. J. Debal fig. 1, 3 et 4, J. Mertens (fig. 2), Lelong (fig. 5), Bailly: fig. 7 . 